\title{
Mitosis-related gene CENP-U as a potential biomarker in malignancy
}

\author{
Changjian Shao ${ }^{1 \#}$, Yuanyong Wang ${ }^{1 \#}$, Hongtao Duan ${ }^{1 \#}$, Peng Ding ${ }^{1}$, Yimeng Zhang ${ }^{2}$, Jiayi Ning ${ }^{2}$, Jing Han ${ }^{2}$, \\ Tao Jiang ${ }^{1}$, Xiaolong Yan ${ }^{1}$ \\ ${ }^{1}$ Department of Thoracic Surgery, Tangdu Hospital, The Fourth Military Medical University, Xi'an, China; ${ }^{2}$ Department of Ophthalmology, Tangdu \\ Hospital, The Fourth Military Medical University, Xi'an, China \\ Contributions: (I) Conception and design: C Shao, Y Wang, H Duan; (II) Administrative support: T Jiang, X Yan; (III) Provision of study materials \\ or patients: J Han, X Yan; (IV) Collection and assembly of data: C Shao, Y Wang, P Ding; (V) Data analysis and interpretation: C Shao, T Jiang, Y \\ Zhang, J Ning; (VI) Manuscript writing: All authors; (VII) Final approval of manuscript: All authors. \\ \#These authors contributed equally to this work. \\ Correspondence to: Jing Han. Department of Ophthalmology, Tangdu Hospital, The Fourth Military Medical University, 1 Xinsi Road, Xi'an 710038, \\ China. Email: hanjing.cn@163.com; Tao Jiang; Xiaolong Yan. Department of Thoracic Surgery, Tangdu Hospital, The Fourth Military Medical \\ University, 1 Xinsi Road, Xi’an 710038, China. Email: jiangtaochest@163.com; yanxiaolong@fmmu.edu.cn.
}

Background: Centromere protein $\mathrm{U}(C E N P-U)$ is a component of the kinetochore and can regulate the cell cycle as a receptor of polo-like kinase 1 (PLK1). Recent studies have partially identified the role of $C E N P-U$ in tumor progression, but the underlying mechanisms of $C E N P-U$ in tumor immunity remain obscure.

Methods: We performed pan-cancer analysis to evaluate the role of $C E N P-U$ in immunity and proliferation with data from The Cancer Genome Atlas (TCGA), Cancer Cell Line Encyclopedia (CCLE) datasets, and Genotype-Tissue Expression (GTEx) project. Results of CENP-U expression and related clinicopathological data were obtained to show the expression levels, prognosis, tumor progression, immune neoantigens, and immune checkpoints of $C E N P-U$ in 33 tumors. The Tumor Immune Estimation Resource (TIMER) dataset was used to analyze immune infiltration scores.

Results: Results of the pan-cancer analysis demonstrated that CENP- $U$ is differentially expressed in normal tissues and common tumor tissues. Moreover, differentially expressed $C E N P-U$ was also identified between matched normal and tumor tissues, and the high expression level of CENP-U was associated with poor prognosis for 33 kinds of tumor except for that of thymoma (THYM) and lymphoid neoplasm diffuse large B-cell lymphoma (DLBC). Furthermore, the correlation between CENP-U expression and immune checkpoints and immune neoantigens was determined. In addition, CENP- $U$ expression was correlated with tumor-infiltrating immune cells especially in THYM but not in lung squamous cell carcinoma (LUSC), esophageal carcinoma (ESCA), or lung adenocarcinoma (LUAD). Finally, gene set enrichment analysis (GSEA) indicated that CENP-U is critically involved in tumor proliferation, immunity, and metabolism.

Conclusions: $C E N P-U$, a mitosis-related kinase, was found to be differentially expressed across different cancer types and to play an important role in tumor progression and immunity. CENP-U holds the potential to be a prognostic marker, whose targeting may provide therapeutic benefit.

Keywords: Centromere protein $\mathrm{U}(C E N P-U)$; mitosis; pan-cancer analysis; immunity

Submitted Nov 26, 2021. Accepted for publication Dec 10, 2021.

doi: 10.21037/atm-21-6516

View this article at: https://dx.doi.org/10.21037/atm-21-6516 


\section{Introduction}

Cancer is a serious public problem and is the leading cause of death worldwide (1). Recently, with the emergence of multimodal therapies, patients with tumor have benefited greatly from chemotherapy, radiotherapy, and particularly immunotherapy, $(2,3)$, but the prognosis of a majority of patients remains poor (4). Therefore, there is a pressing need for more effective therapies to be developed for cancer therapy. Cancer cells are characterized by a loss of mitotic regulation, which may further result in unlimited replicative potential and aberrant proliferation $(5,6)$ In the process of mitosis, both the centromere and kinetochore play important roles in accurately distributing replicated genome (7).

Equal distribution of DNA in mitosis requires the centromere, a region of chromosome, and a large amount of proteins assembled onto the centromeric DNA, which is called the kinetochore. In most instances, centromere specification is dependent on the presence of centromere protein $\mathrm{A}(C E N P-A)$, a variant of histone $\mathrm{H} 3$ (core submit of nucleosomes). Moreover, 16 proteins that constitute the centromere-associated network (CCAN) are assembled onto centromeric CENP-A: CENP-O/CENP-P/CENP-Q/ CENP-R/CENP-U (CENP-50), CENP-C, CENP-H/ CENP-I/CENP-K, CENP-L/CENP-M/CENP-N, CENP-S/ $C E N P-X$, and CENP-T/CENP- $W$. These proteins form the inner kinetochore and can further recruit components of the outer kinetochore (8). As an essential component of the CCAN, CENP-U was recently reported to act as a tumor promoter (9).

Centromere protein $\mathrm{U}(C E N P-U)$, also known as CENP50, polo-box-interacting protein 1 (PBIP1), Kaposi's sarcoma-associated herpes virus latent nuclear antigeninteracting protein 1 (KLIP1), or myeloid leukemia factor 1-interacting protein $(M L F 1 I P)$, belongs to the CENP-O complex of CCAN, which includes CENP-O, CENP-P, $C E N P-Q, C E N P-R$, and $C E N P-U$. For one, CENP-U is a part of the kinetochore, and its deficiency was reported to lead to embryonic lethality in mice and Holstein cattle. Additionally, disrupted CENP-U expression of mouse ES cells was found to result in the disappearance of all CENP-O class proteins and ultimately mitotic defects $(10,11)$, further demonstrating the importance of CENP- $U$ in mitosis. Moreover, PBIP1 (CENP-U) is essential for recruiting polo-like kinase 1 (PLK1) to the kinetochore and may be the sole receptor of $P L K 1$ in core kinetochore, which is crucial for promoting centrosome maturation and spindle assembly $(12,13)$. That is, centromere proteins are not only indispensable components of kinetochore but also act as a vital role in protecting kinetochore-microtubule interface, which ensures the accurate distribution of chromosome. Intriguingly, $C E N P-U$ plus $C E N P-A, C E N P-N$, and $C E N P-T$ was reported to be rapidly recruited to where double-strand breaks are present in DNA, which indicates that CENP-U may play a role in DNA damage repair (DDR) (14). For another, CENP-I was recently reported to promote the proliferation and migration of gastric cancer (GC) and a series of CENP proteins including $C E N P-U$ were reported to be upregulated in GC (15). As an important regulator of mitosis, $C E N P-U$ was found to be elevated in breast cancer (BRCA) (16,17), glioblastoma multiforme (GBM) (18), prostate adenocarcinoma (PRAD) (19), osteosarcoma (20), liver hepatocellular carcinoma (LIHC) (21), bladder urothelial carcinoma (BLCA) (22), lung adenocarcinoma (LUAD) (23), and ovarian cancer (OV) (24); further, highly expressed $C E N P-U$ has been correlated with poor prognosis (16,17,21-23). However, the underlying mechanisms of $C E N P-U$ in tumor immunity, metabolism, and proliferation are not fully understood, and thus further analysis is needed.

In this study, the expression of $C E N P-U$ and its potential pan-cancer prognostic value was analyzed with The Cancer Genome Atlas (TCGA), Genotype-Tissue Expression (GTEx), and Cancer Cell Line Encyclopedia (CCLE) datasets. To date, immune checkpoint inhibitors (ICIs) have become important weapons in fighting cancer, which could eliminate the immune suppressive effect of innate and adaptive immune cells (25). At the same time, biomarkers that could predict efficacy of ICIs, such as tumor mutational burden (TMB) and microsatellite instability (MSI), are also needed for non-responsiveness of ICIs in some cases $(26,27)$. Thus, correlation analysis between $C E N P-U$ expression level and immune checkpoints, tumor-infiltrating immune cells, TMB, and MSI was performed. Finally, we performed gene set enrichment analysis (GSEA) analysis to identify the signaling pathways linked to $C E N P-U$. Taken together, our pan-cancer analyses provide insights into the therapeutic and prognostic role of $C E N P-U$ in common cancers.

We present the following article in accordance with the REMARK reporting checklist (available at https://dx.doi. org/10.21037/atm-21-6516).

\section{Methods}

\section{Sample source}

First, CENP-U expression data of tumor cell lines were 
downloaded from the CCLE database (https://portals. broadinstitute.org/ccle) (28). Moreover, we collected data from RNA sequencing in normal and tumor tissues from the GTEx dataset (https://commonfund.nih.gov/GTEx/) and TCGA database (https://portal.gdc.cancer.gov), respectively. Data of $C E N P-U$ expression were obtained from the two datasets, and the related clinicopathological data were also acquired. Additionally, cancer immune infiltration scores were analyzed with data from the Tumor Immune Estimation Resource (TIMER) dataset (29). The study was conducted in accordance with the Declaration of Helsinki (as revised in 2013).

\section{Expression analysis of CENP-U}

We analyzed the expression of $C E N P-U$ in normal tissues and cancer cell lines. In addition, the Kruskal-Wallis test was used to analyze the differential expression between normal and tumor tissues with data solely from TCGA database. Following this, CENP-U expression data in normal tissues from the GTEx dataset were compared with the tumor data from TCGA database.

\section{Correlation analysis of CENP-U expression level and prognosis in pan-cancer}

To confirm the pan-cancer prognostic role of $C E N P-U$, survival analysis and univariate Cox proportional hazards regression analysis were used. Prognostic indicators in this study consisted of overall survival (OS) rate, disease-free interval (DFI), progression-free interval (PFI), and diseasespecific survival (DSS). Survival curves and forest plots were obtained.

\section{Correlation analysis of CENP-U expression level and immunity}

Immune checkpoints, immune neoantigens, and the tumor microenvironment (TME) were analyzed. First, Spearman's rank correlation coefficient was performed to analyze the association between $C E N P-U$ expression and immune checkpoints, which include chemokine receptor proteins, chemokine, immune activation proteins, and immunosuppressive proteins. Similar analyses were applied to evaluate the correlation of $C E N P-U$ expression and immune neoantigens and tumor-infiltrating immune cells, including $\mathrm{B}$ cells, $\mathrm{CD}^{+} \mathrm{T}$ cells, $\mathrm{CD} 8^{+} \mathrm{T}$ cells, neutrophils, macrophages, and dendritic cells (DC). Furthermore, immune core, stromal score, and ESTIMATE score were obtained with the Estimation of Stromal and Immune Cells in Malignant Tumors Using Expression data (ESTIMATE) algorithm (30).

\section{Correlation analysis of CENP-U expression level and TMB and MSI}

Pearson correlation coefficient was employed to explore the association of $C E N P-U$ expression and TMB and MSI. Bubble charts were obtained.

\section{GSEA}

Enriched signaling pathways in $C E N P-U$ higher and lower expression were analyzed using GSEA. The Hallmark gene set and the Kyoto Encyclopedia of Genes and Genomes database (KEGG; https://www.kegg.jp.) were applied. A normalized enrichment score (NES) $>1.5$, a $\mathrm{P}$ value $<0.05$, and a false discovery rate (FDR) $<0.25$ were considered significant.

\section{Statistical analysis}

R software (version 4.0.2; https://www.R-project.org; The R Foundation for Statistical Computing, Vienna, Austria) was used in this study to manage data, and the plots were obtained using $\mathrm{R}$ packages. A $\mathrm{P}$ value $<0.05$ was considered to be statistically significant.

\section{Results}

\section{Differential expression of CENP-U}

Data from the CCLE database, GTEx dataset, and TCGA database were analyzed to evaluate the relative $C E N P-U$ expression in normal and tumor tissues. Data from the GTEx dataset showed $C E N P-U$ to be commonly expressed in 31 normal tissues, with the highest expression levels present in bone marrow and the testes, and the lowest expression levels in muscle and the heart (Figure 1A). The CCLE data revealed that $C E N P-U$ had an average expression in tumor cell lines (Figure $1 B$ ). By analyzing the expression data of $C E N P-U$ in 26 matched normal and tumor tissues solely from TCGA database, we found that the expression of $C E N P-U$ was elevated in all 26 matched different tumor tissues (Figure 1C). Additionally, CENP-U expression data of matched normal and tumor tissues from 


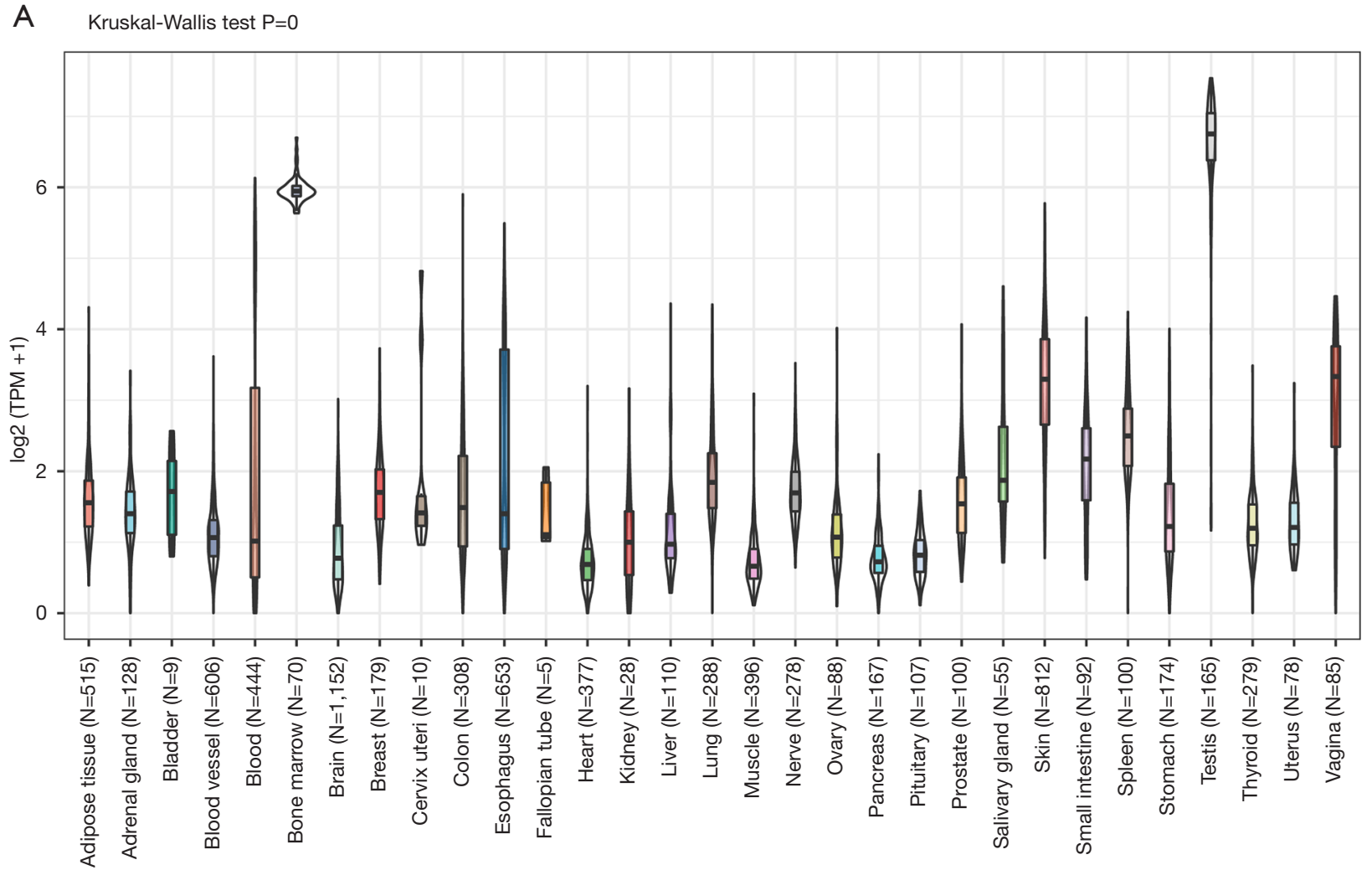

B Kruskal-Wallis test $\mathrm{P}=2.2 \mathrm{e}-18$

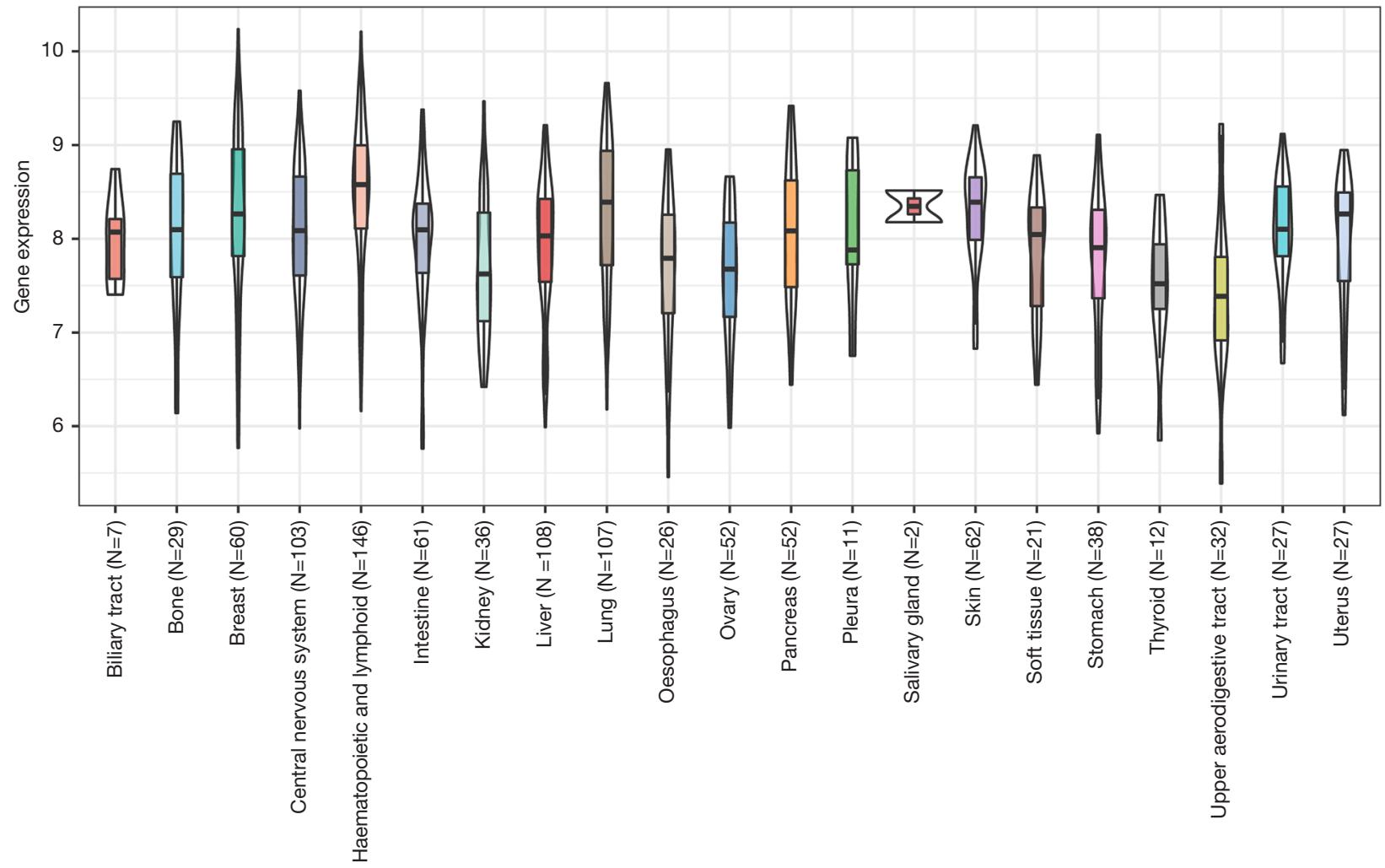



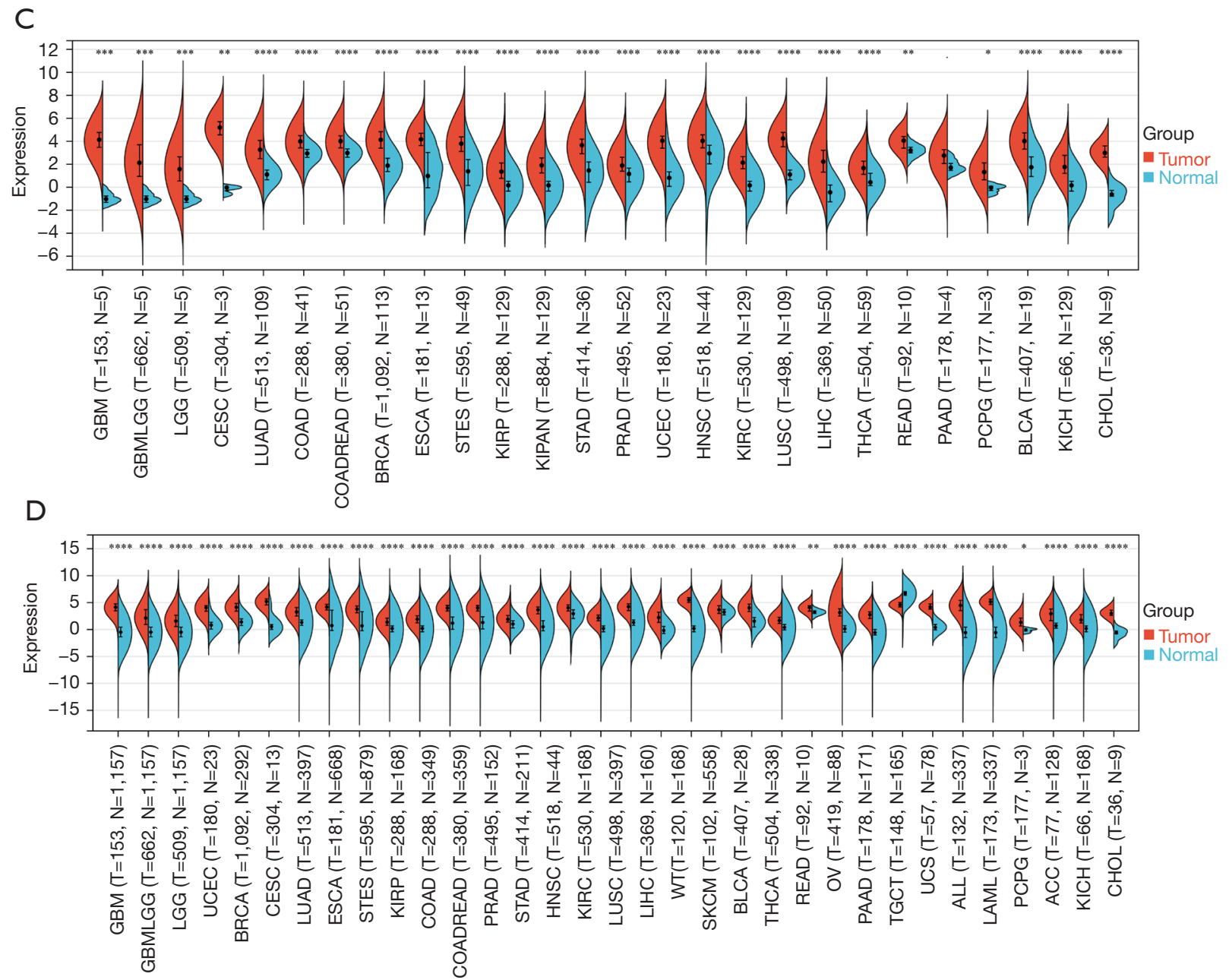

Figure 1 Expression analysis of CENP-U. (A) CENP-U expression level in 31 kinds of normal tissues with data from the GTEx database. (B) $C E N P-U$ expression level in tumor cell lines of 21 kinds of tumors with data from the CCLE dataset. (C) $C E N P-U$ expression level in 26 types of matched tumor and normal tissues with data from TCGA database. (D) CENP-U expression level in 34 types of matched tumor tissues from TCGA database and normal tissues from the GTEx database. ${ }^{*} \mathrm{P}<0.05 ;{ }^{* *} \mathrm{P}<0.01 ;{ }^{* * *} \mathrm{P}<0.001 ;{ }^{* * * *} \mathrm{P}<0.0001 . C E N P-U$, centromere protein U; GTEx, Genotype-Tissue Expression; CCLE, Cancer Cell Line Encyclopedia; TCGA, The Cancer, Genome Atlas.

both the GTEx dataset and TCGA database demonstrated that $C E N P-U$ expression was elevated in GBM, glioma (GBMLGG), brain lower grade glioma (LGG), uterine corpus endometrial carcinoma (UCEC), breast invasive carcinoma (BRCA), cervical squamous cell carcinoma and endocervical adenocarcinoma (CESC), LUAD, esophageal carcinoma (ESCA), stomach and esophageal carcinoma (STES), kidney renal papillary cell carcinoma (KIRP), pankidney cohort (KIPAN), colon adenocarcinoma (COAD), head and neck squamous cell carcinoma (HNSC), colon adenocarcinoma/rectum adenocarcinoma (COADREAD), PRAD, stomach adenocarcinoma (STAD), kidney renal clear cell carcinoma (KIRC), lung squamous cell carcinoma (LUSC), LIHC, Wilms tumor (WT), skin cutaneous melanoma (SKCM), BLCA, thyroid cancer (THCA), rectum adenocarcinoma (READ), OV, pancreatic adenocarcinoma (PAAD), uterine carcinoma (UCS), acute myeloid leukemia (LAML), acute lymphoblastic leukemia (ALL), pheochromocytoma and paraganglioma (PCPG), adrenocortical carcinoma (ACC), kidney chromophobe $(\mathrm{KICH})$, and cholangiocarcinoma (CHOL) (Figure 1D). However, CENP-U expression was not elevated in testicular germ cell tumors (TGCT). These results confirmed $C E N P-U$ expression in tumor tissues to be elevated 
compared to normal tissues among almost all examined cancer types, except for TGCT.

\section{Correlation of CENP-U expression and prognosis}

Univariate Cox proportional hazards regression analysis was performed to assess the pan-cancer prognostic role of $C E N P-U$. Results showed that $C E N P-U$ expression was markedly correlated with OS of patients with LUAD $(\mathrm{P}<0.01)$, GBMLGG $(\mathrm{P}<0.01)$, LGG $(\mathrm{P}<0.01)$, KIPAN $(\mathrm{P}<0.01)$, ACC $(\mathrm{P}<0.01)$, KIRP $(\mathrm{P}<0.01)$, PAAD $(\mathrm{P}<0.01)$, LAML $(\mathrm{P}<0.01)$, KICH $(\mathrm{P}<0.01)$, LIHC $(\mathrm{P}<0.01)$, mesothelioma (MESO) $(\mathrm{P}<0.01)$, lymphoid neoplasm diffuse large B-cell lymphoma (DLBC) $(\mathrm{P}<0.01)$, CHOL $(\mathrm{P}=0.04)$, TGCT $(\mathrm{P}=0.03)$, and thymoma (THYM) $(\mathrm{P}=0.04)$ (Figure $2 A$ ). In addition, results of DSS revealed that $C E N P-U$ expression correlated to the DSS of patients with GBMLGG $(\mathrm{P}<0.01)$, KIPAN $(\mathrm{P}<0.01)$, LGG $(\mathrm{P}<0.01)$, KIRP $(\mathrm{P}<0.01)$, ACC $(\mathrm{P}<0.01)$, KICH $(\mathrm{P}<0.01)$, LUAD $(\mathrm{P}<0.01), \mathrm{PAAD}(\mathrm{P}<0.01)$, MESO $(\mathrm{P}<0.01), \mathrm{PRAD}(\mathrm{P}<0.01)$, SKCM $(\mathrm{P}<0.01)$, LIHC $(\mathrm{P}=0.01)$, KIRC $(\mathrm{P}=0.03)$, and $\mathrm{OV}(\mathrm{P}=0.03)$ (Figure $2 B)$. Moreover, the expression level of $C E N P-U$ was associated with the PFI of patients with GBMLGG $(\mathrm{P}<0.01)$, LGG $(\mathrm{P}<0.01)$, KIRP $(\mathrm{P}<0.01)$, ACC $(\mathrm{P}<0.01)$, KIPAN $(\mathrm{P}<0.01)$, LIHC $(\mathrm{P}<0.01)$, PRAD $(\mathrm{P}<0.01)$, KICH $(\mathrm{P}<0.01)$, PAAD $(\mathrm{P}<0.01)$, uveal melanoma (UVM) $(\mathrm{P}<0.01)$, LUAD $(\mathrm{P}<0.01)$, MESO $(\mathrm{P}<0.01)$, SKCM $(\mathrm{P}=0.02)$, and BLCA $(\mathrm{P}=0.04)$ (Figure $2 C)$. We further found that the expression $C E N P-U$ to be significantly linked to the DFI of patients with KIRP $(\mathrm{P}<0.01)$, LIHC $(\mathrm{P}<0.01)$, THCA $(\mathrm{P}<0.01)$, sarcoma (SARC) $(\mathrm{P}=0.02)$, MESO $(\mathrm{P}=0.02)$, and $\mathrm{PAAD}(\mathrm{P}=0.04)$ (Figure $2 D)$.

\section{Prognostic role of CENP-U in pan-cancer}

$C E N P-U$ was grouped into a high expression and low expression group, with the subsequent survival analysis indicating high $C E N P-U$ expression to be associated with shorter OS in patients with ACC, KICH, KIRC, KIRP, LGG, LIHC, LUAD, MESO, PAAD, PCPG, PRAD, and UVM; and high $C E N P-U$ expression to be associated with longer OS in patients THYM and DLBC (Figure 3). Results of DSS indicated high CENP- $U$ expression to be correlated with a poor prognosis in patients with ACC, BLCA, KICH, KIRC, KIRP, LGG, LIHC, LUAD, MESO, PAAD, PCPG, PRAD, and UVM (Figure 4). Moreover, higher CENP-U expression was associated with shorter PFI in patients with ACC, BLCA, KICH, KIRC, KIRP, LGG, LIHC, LUAD,
MESO, PAAD, PCPG, PRAD, SARC, THCA, and UVM (Figure 5). Similarly, higher $C E N P-U$ expression was found to be linked to poor DFI in patients with KIRP, LIHC, MESO, SARC, and THCA (Figure 6).

\section{Correlation of CENP-U expression and immunity}

To explore the role of $C E N P-U$ in tumor immunity; data of immune checkpoints; neoantigens, tumor-infiltrating immune cells; and immune score, stromal score, and ESTIMATE score were analyzed. We first performed correlation analysis of $C E N P-U$ expression and immune checkpoints, which included 24 immune inhibitors and 36 immune stimulators. Among the data of immune inhibitors in 40 common tumors, we found that CENP-U expression was positively linked to vascular endothelial growth factor A (VEGFA) in 21 tumors, to CD276 in 25 tumors, to lymphocyte activating 3 (LAG3) in 21 tumors, and to programmed death ligand 1 (PD-L1) in 21 tumors. Additionally, among the data of immune stimulators, $C E N P-U$ expression was found to be positively associated with high mobility group box 2 (HMGB2) in all 40 tumors, C-X-C motif chemokine ligand 10 (CXCL10) in 22 tumors, C-X-C motif chemokine ligand 9 (CXCL9) in 21 tumors (positively), and tumor necrosis factor superfamily member 4 (TNFSF4) in 20 tumors; conversely, CENP- $U$ expression was found to be negatively associated with selectin P (SELP) in 20 tumors. Moreover, CENP- $U$ expression was positively associated with 17 of 24 immune inhibitors and with 24 of 36 immune stimulators in UVM, 22 of 24 inhibitors and 35 of 36 stimulators in OV, 21 of 24 inhibitors and 33 of 36 stimulators in LIHC, 20 of 24 inhibitors and 28 of 36 stimulators in KIPAN, 10 of 24 inhibitors and 23 of 36 stimulators in PAAD, and 0 of 24 inhibitors and 1 of 36 stimulators in UCS (Figure 7A). Next, results of neoantigen analysis suggested that $C E N P-U$ expression was positively associated with the number of neoantigens in BRCA, STAD, PRAD, and LGG (Figure $7 B$ ). Moreover, correlation analysis of $C E N P-U$ expression and 6 types of immune cells of the TME, which consist of $\mathrm{B}$ cells, $\mathrm{CD}^{+} \mathrm{T}$ cells, $\mathrm{CD}^{+} \mathrm{T}$ cells, neutrophils, macrophages, and DCs, was performed (31). Results showed that in THYM, significantly positive correlations were identified between $C E N P-U$ expression and $\mathrm{B}$ cells, $\mathrm{CD} 4^{+} \mathrm{T}$ cells, $\mathrm{CD} 8^{+} \mathrm{T}$ cells, and DCs. In addition, $C E N P-U$ expression was positively associated with neutrophils in KIRC, $\mathrm{CD} 8^{+} \mathrm{T}$ cells in GBMLGG, and DCs in PAAD, but negatively associated with $\mathrm{CD}^{+}{ }^{+} \mathrm{T}$ cells in UVM, DC and neutrophils in TGCT, 
A

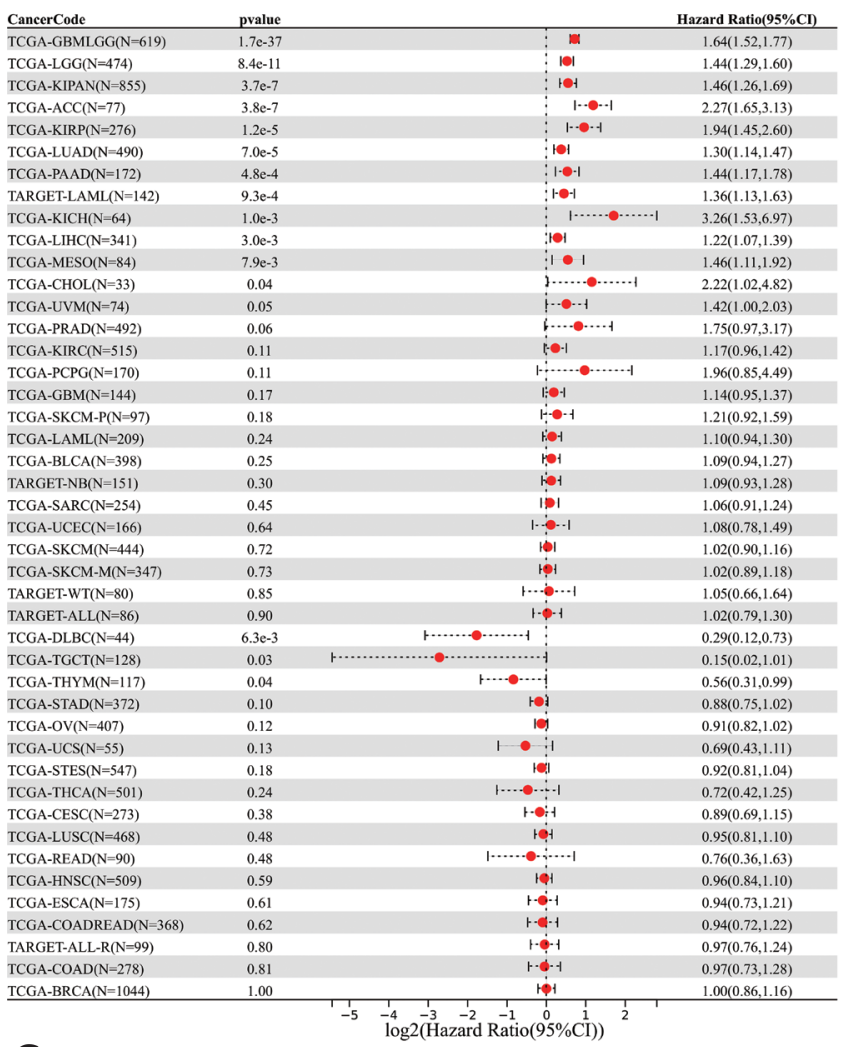

C

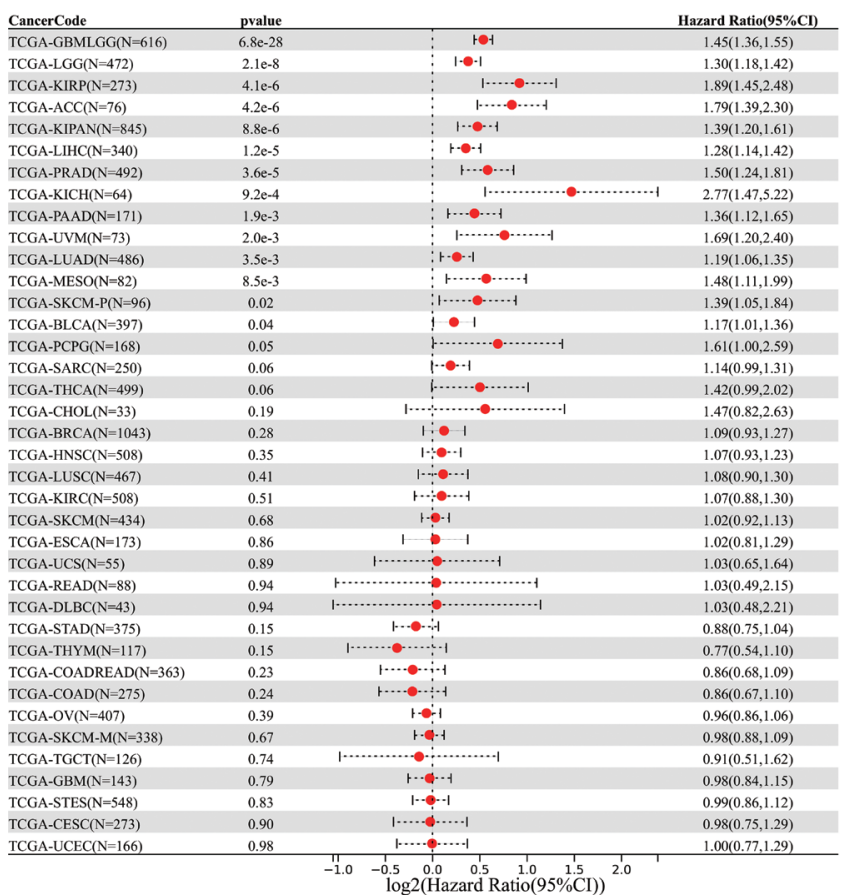

B

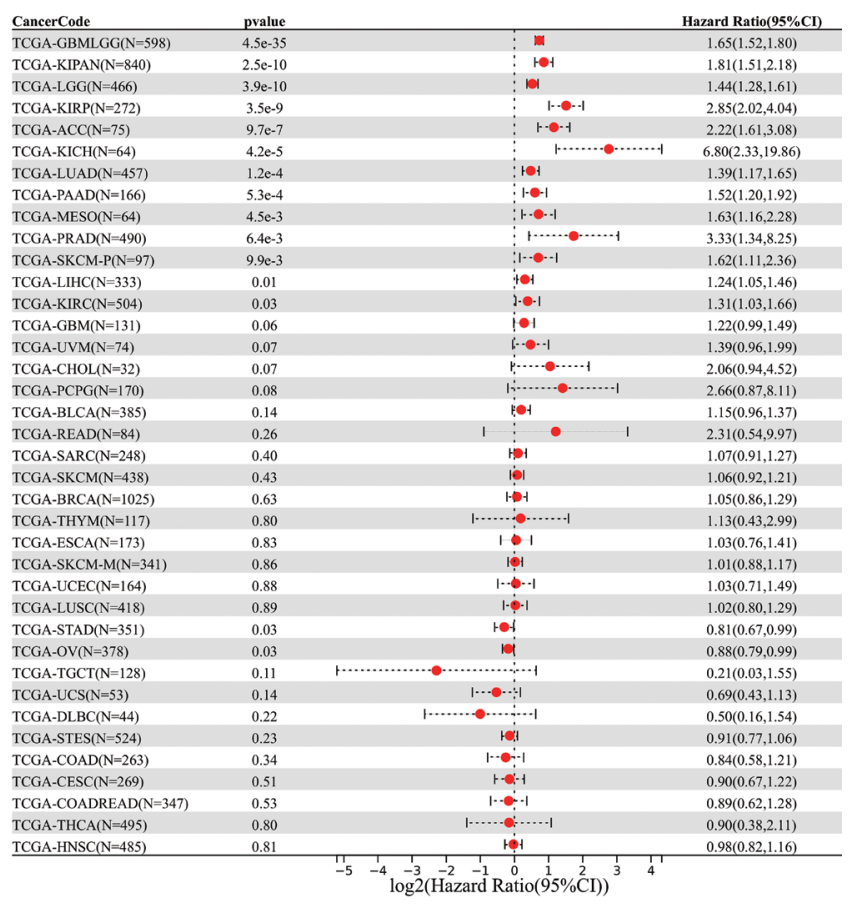

D

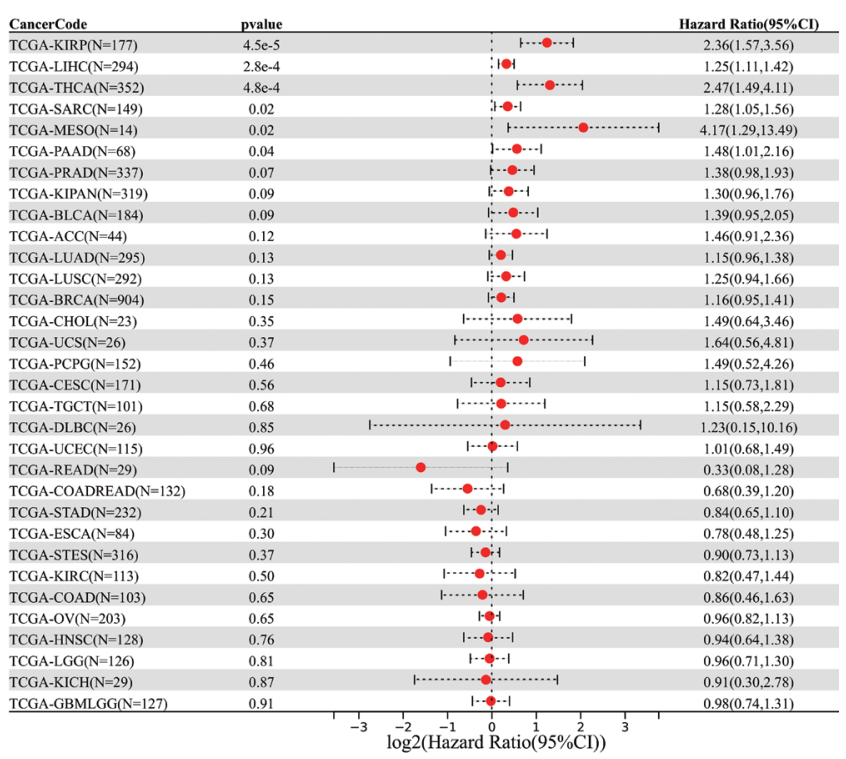

Figure 2 Associations between CENP-U expression and OS, DSS, DFI, and PFI. (A-D) Results of correlation analysis of $C E N P-U$ expression with OS, DSS, PFI, and DFI. Data are shown as forest plots. CENP-U, centromere protein U; OS, overall survival; DSS, diseasespecific survival; DFI, disease-free interval; PFI, progression-free interval. 
A
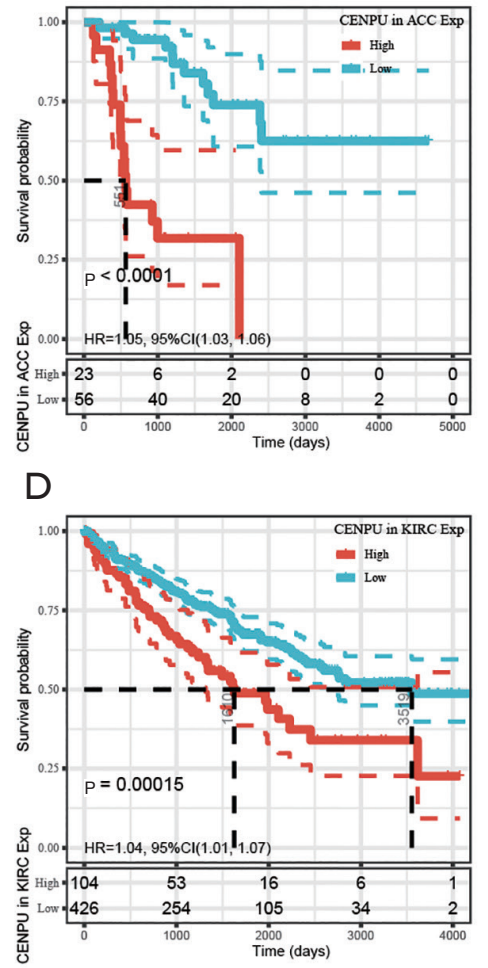

G

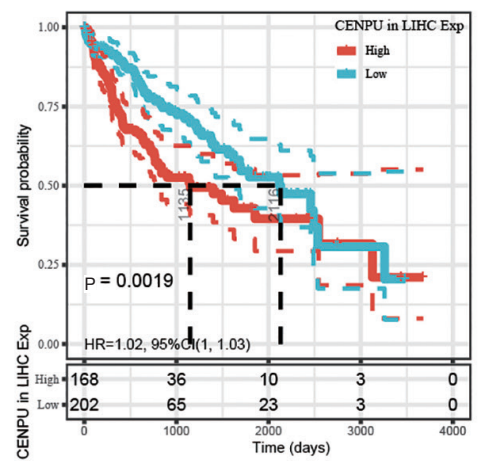

J

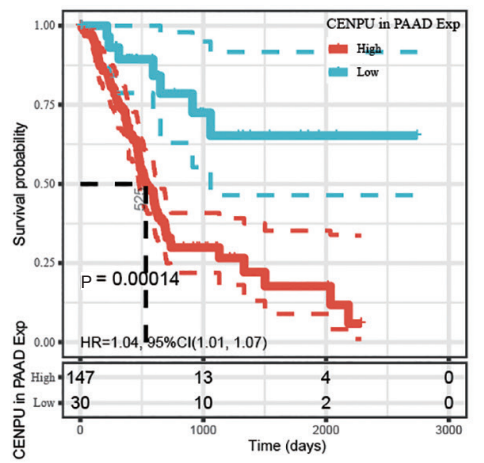

B

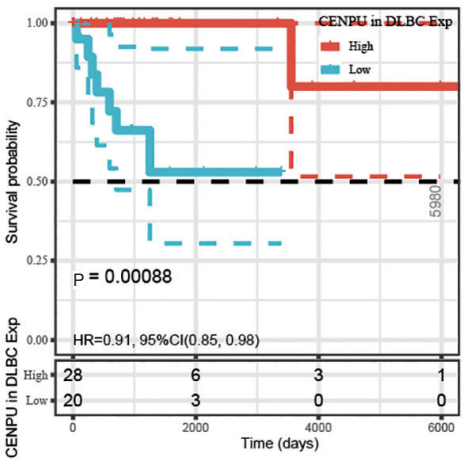

E
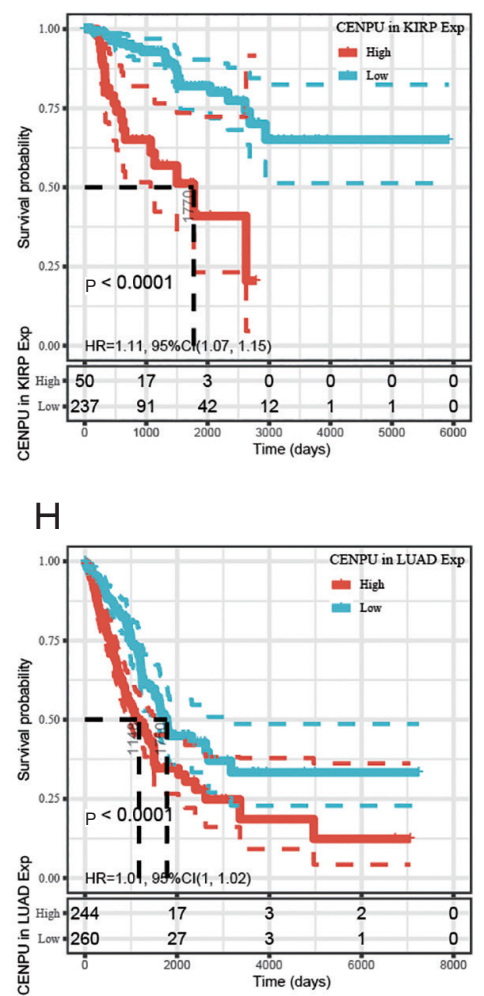

K

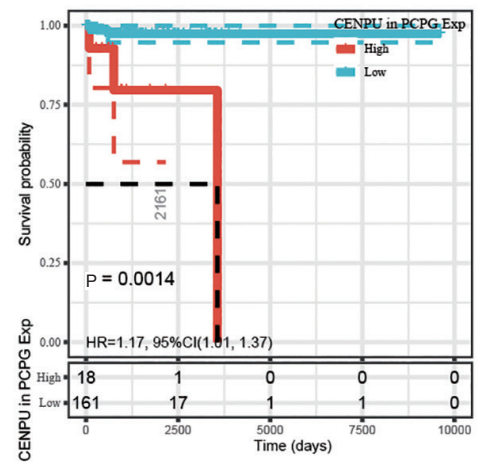

C
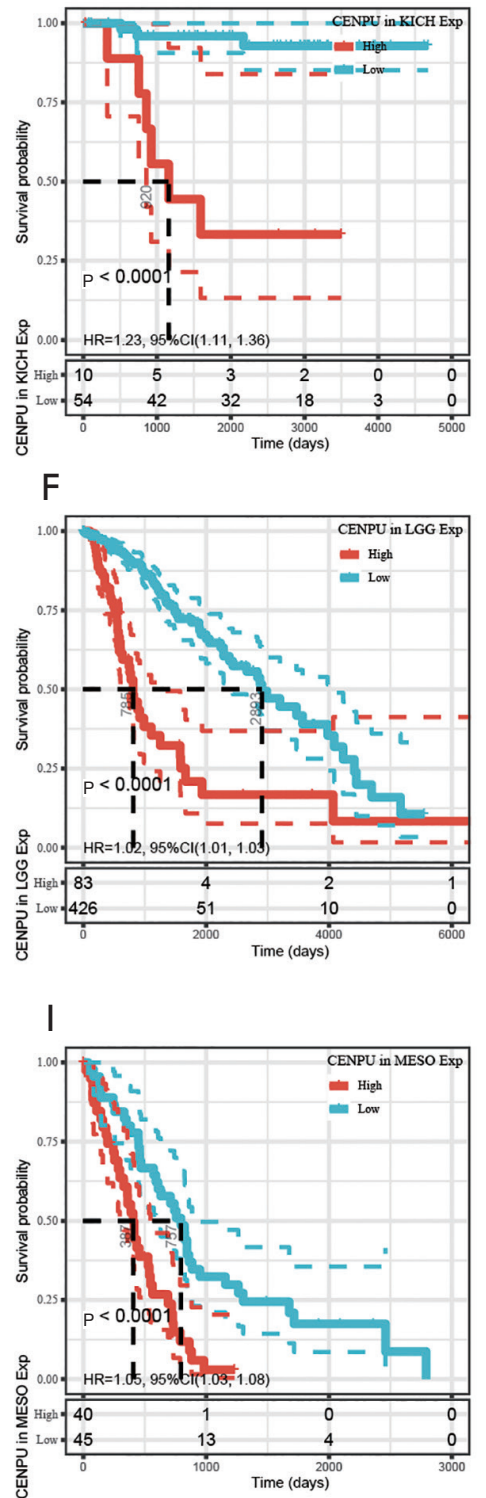

$\mathrm{L}$

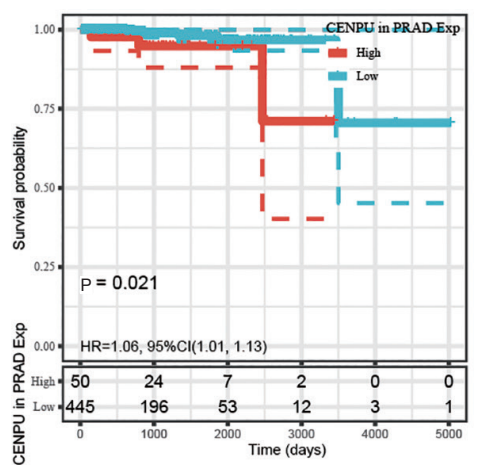


M

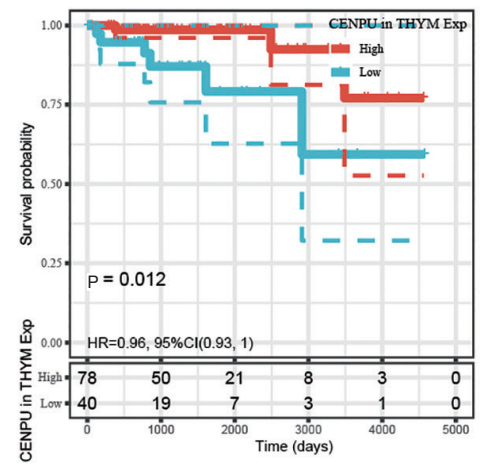

$\mathrm{N}$

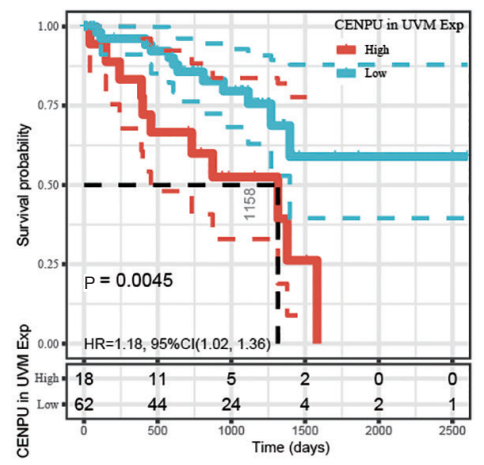

Figure $3 \mathrm{KM}$ analysis between CENP-U expression and OS in ACC, KICH, KIRC, KIRP, LGG, LIHC, LUAD, MESO, PAAD, PCPG, PRAD, UVM, THYM and DLBC. Data are shown with KM curves. KM, Kaplan-Meier; CENP-U, centromere protein U; OS, overall survival; ACC, adrenocortical carcinoma; KICH, kidney chromophobe; KIRC, kidney renal clear cell carcinoma; KIRP, kidney renal papillary cell carcinoma; LGG, brain lower grade glioma; LIHC, liver hepatocellular carcinoma; LUAD, lung adenocarcinoma; MESO, mesothelioma; PAAD, pancreatic adenocarcinoma; PCPG, pheochromocytoma and paraganglioma; PRAD, prostate adenocarcinoma; UVM, uveal melanoma; THYM, thymoma; DLBC, lymphoid neoplasm diffuse large B-cell lymphoma.

A

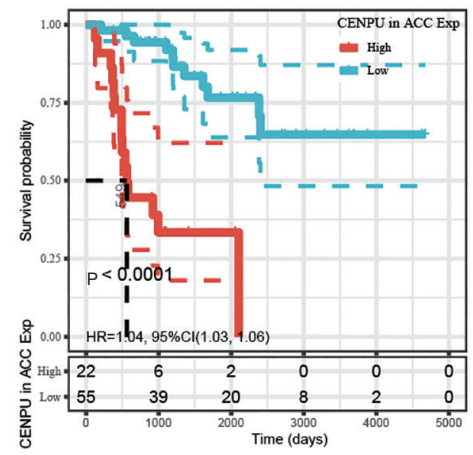

D

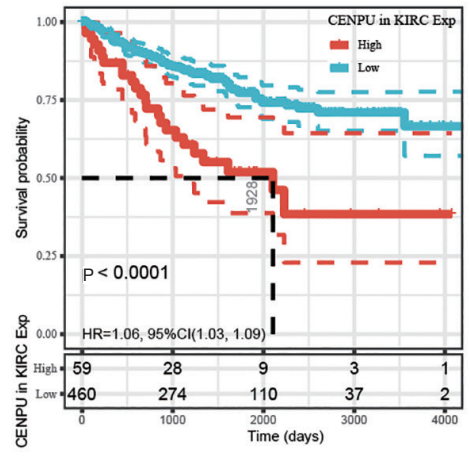

B

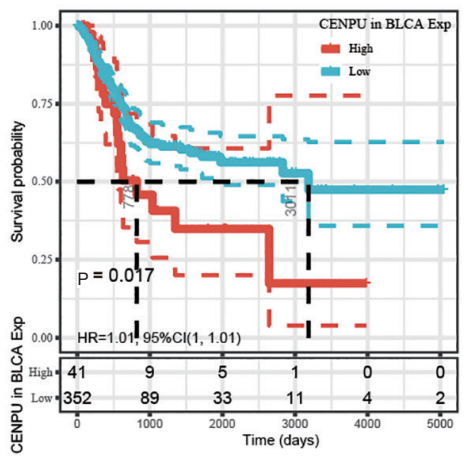

E

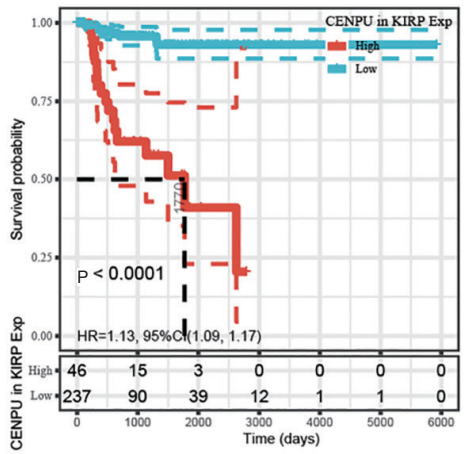

C

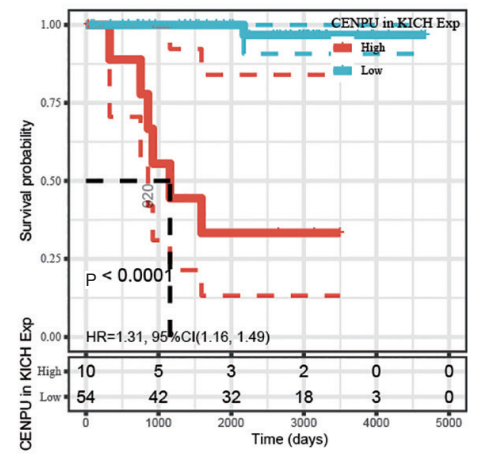

F

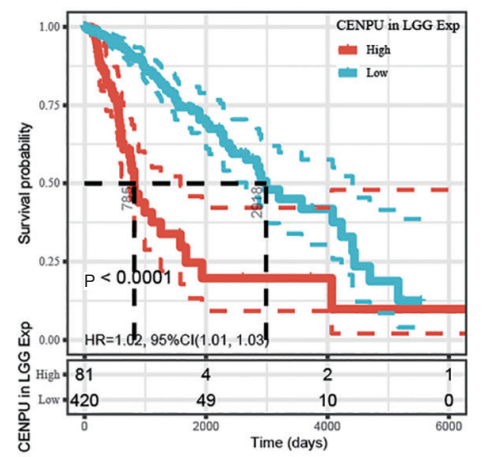



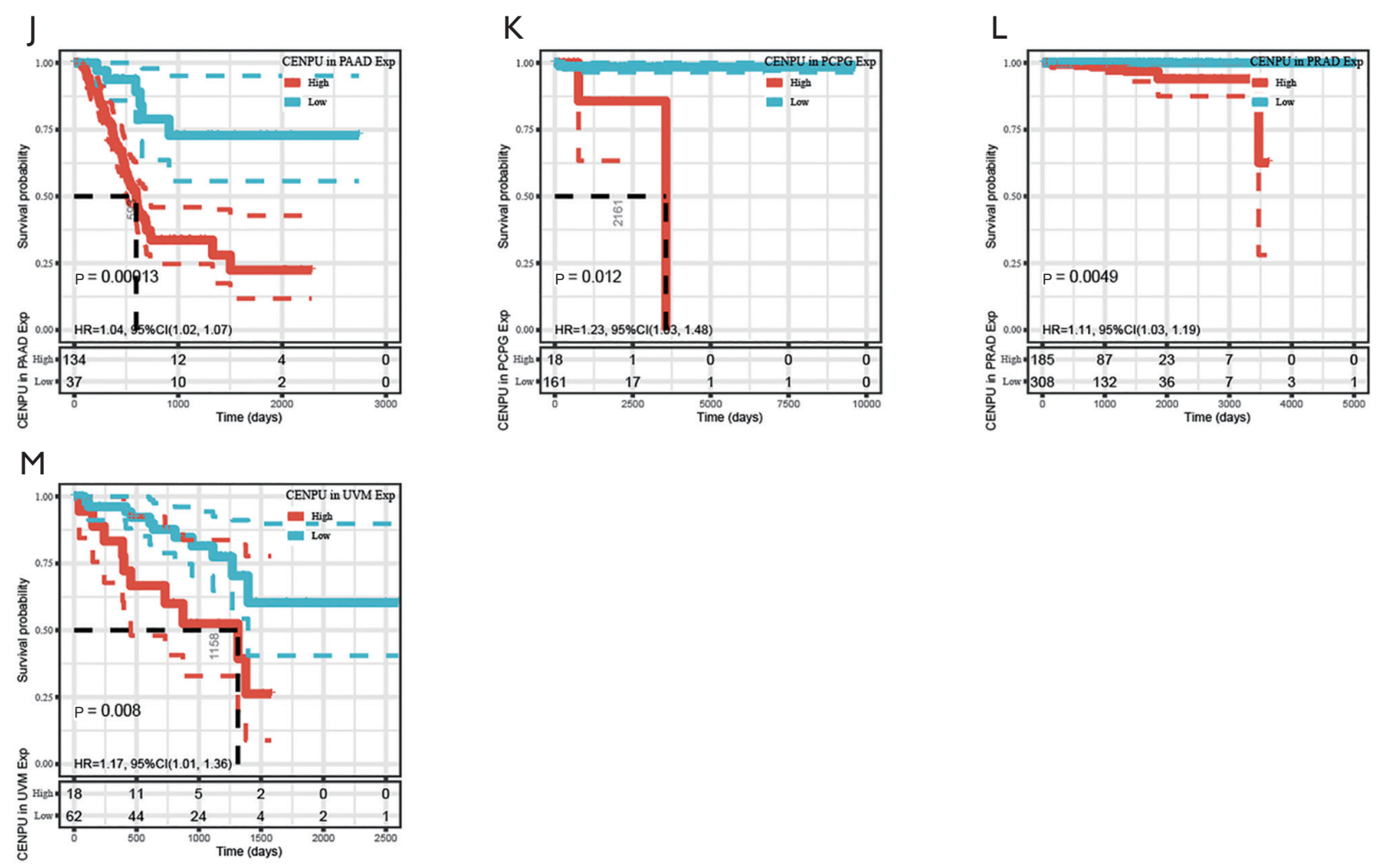

Figure $4 \mathrm{KM}$ analysis between $C E N P-U$ expression and DSS in ACC, BLCA, KICH, KIRC, KIRP, LGG, LIHC, LUAD, MESO, PAAD, PCPG, PRAD, and UVM. Data are shown with KM curves. KM, Kaplan-Meier; CENP-U, centromere protein U; DSS, disease-specific survival; ACC, adrenocortical carcinoma; BLCA, bladder urothelial carcinoma; KICH, kidney chromophobe; KIRC, kidney renal clear cell carcinoma; KIRP, kidney renal papillary cell carcinoma; LGG, brain lower grade glioma; LIHC, liver hepatocellular carcinoma; LUAD, lung adenocarcinoma; MESO, mesothelioma; PAAD, pancreatic adenocarcinoma; PCPG, pheochromocytoma and paraganglioma; PRAD, prostate adenocarcinoma; UVM, uveal melanoma.

$\mathrm{CD}^{+} \mathrm{T}$ cells in DLBC, and DCs in UVM (Figure $8 A$ ). Finally, data of ESTIMATE analysis revealed that CENP-U expression was negatively correlated with stromal score in BRCA, STES and STAD (Figure 8B).

\section{Correlation of CENP-U expression and TMB and MSI}

Cancer is a kind of genome disease, which could be caused by accumulations of point mutations and structural alterations. TMB and MSI may be comprehensive indicators to reflect the level of genomic instability (32). In this study, we found that $C E N P-U$ expression was positively correlated to TMB in PCPG, ACC, COADREAD, STAD, COAD and LUAD (Figure 9A). Similarly, CENP-U expression was found to be associated with MSI in GBMLGG (negatively), DLBC (negatively), STAD (positively), COADREAD (positively), READ (positively) and COAD (positively) (Figure 9B).

\section{GSEA}

Protein-protein interaction (PPI) network analysis was employed to clarify the functional network of $C E N P-U$ in order to fully understand the relevant mechanisms. Results demonstrated that $C E N P-U$ was linked to CENP-O, CENP-P, CENP-Q, CENP-R, CENP-I, CENP-H, CENP-M, $C E N P-N, C E N P-T$, and $P L K 1$, all of which are mitosisrelated proteins (Figure 10A). According to the median expression level of $C E N P-U$, high expression and low expression groups were formed and then analyzed by GSEA. The resulting data indicated $C E N P-U$ to be highly enriched in Hallmark DNA repair (Figure 10B), Hallmark 
A

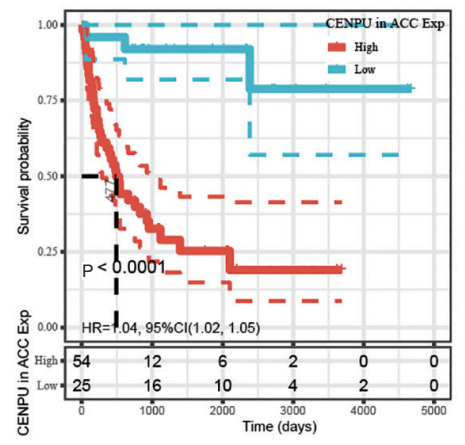

D

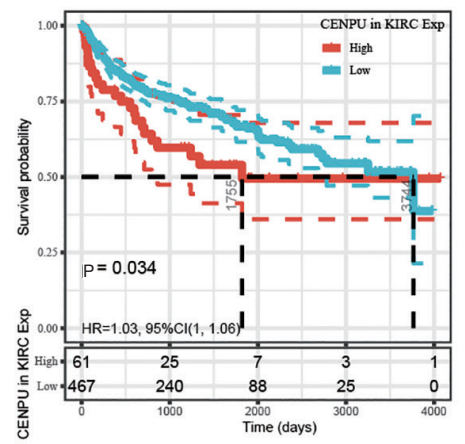

G

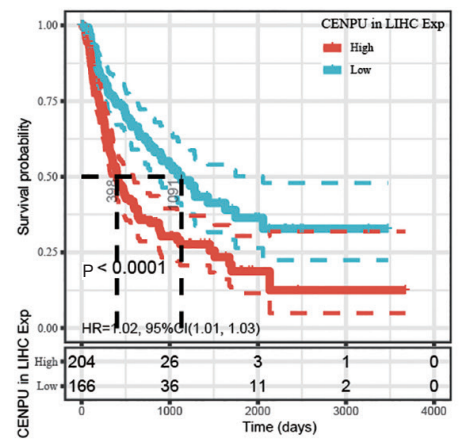

J

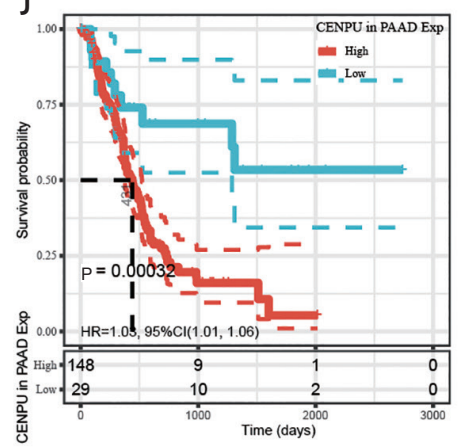

B

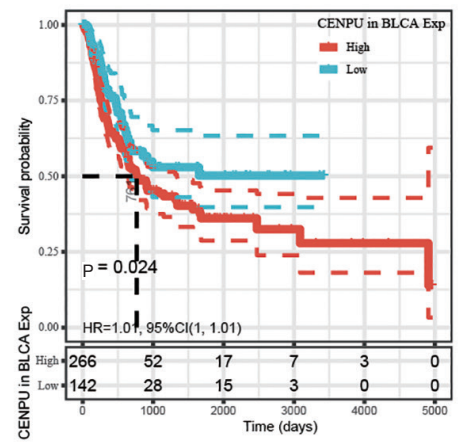

E

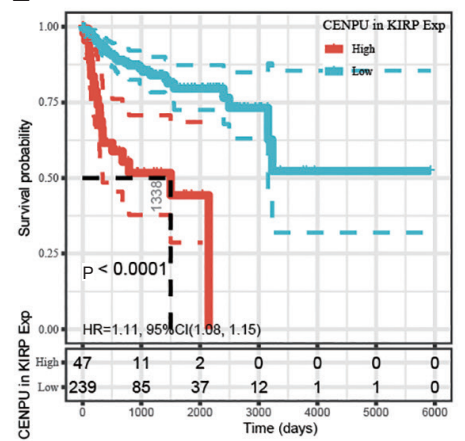

$\mathrm{H}$

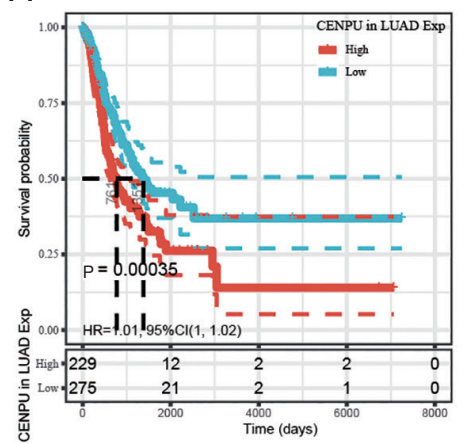

$\mathrm{K}$

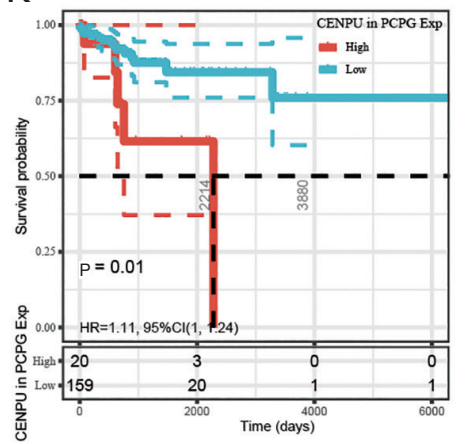

C

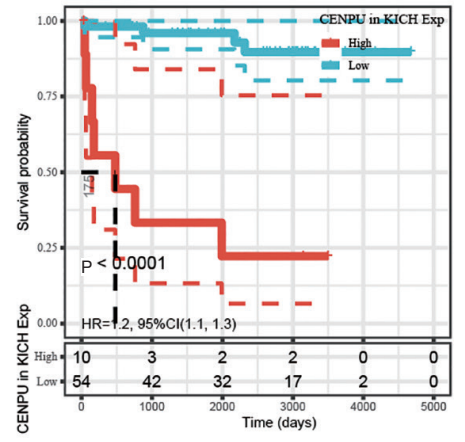

$\mathrm{F}$

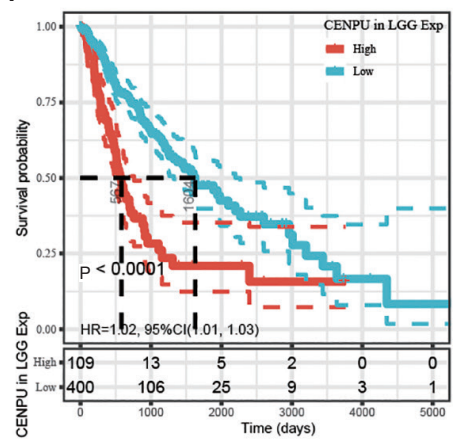

I

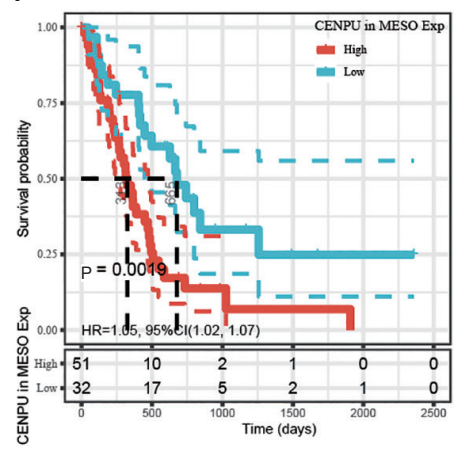

$\mathrm{L}$

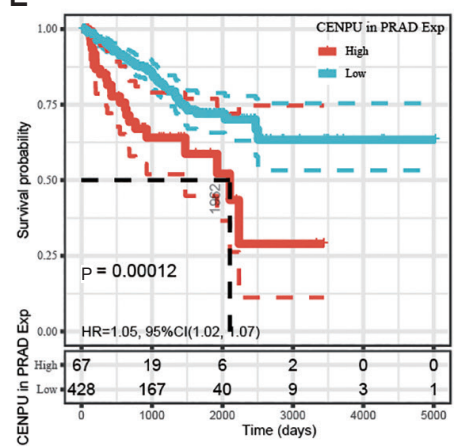


M

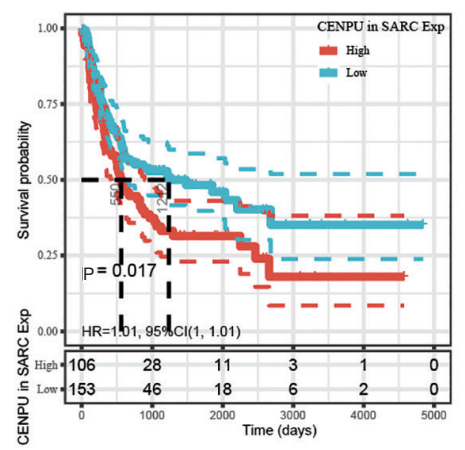

$\mathrm{N}$

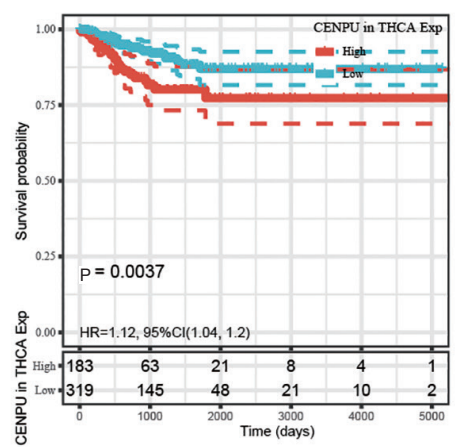

O

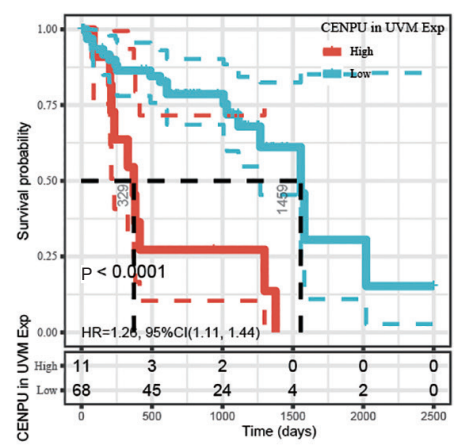

Figure $5 \mathrm{KM}$ analysis between $C E N P-U$ expression and PFI in ACC, BLCA, KICH, KIRC, KIRP, LGG, LIHC, LUAD, MESO, PAAD, PCPG, PRAD, SARC, THCA, and UVM. Data are shown with KM curves. KM, Kaplan-Meier; CENP-U, centromere protein U; PFI, progression-free interval; ACC, adrenocortical carcinoma; BLCA, bladder urothelial carcinoma; KICH, kidney chromophobe; KIRC, kidney renal clear cell carcinoma; KIRP, kidney renal papillary cell carcinoma; LGG, brain lower grade glioma; LIHC, liver hepatocellular carcinoma; LUAD, lung adenocarcinoma; MESO, mesothelioma; PAAD, pancreatic adenocarcinoma; PCPG, pheochromocytoma and paraganglioma; PRAD, prostate adenocarcinoma; SARC, sarcoma; THCA, thyroid cancer; UVM, uveal melanoma.
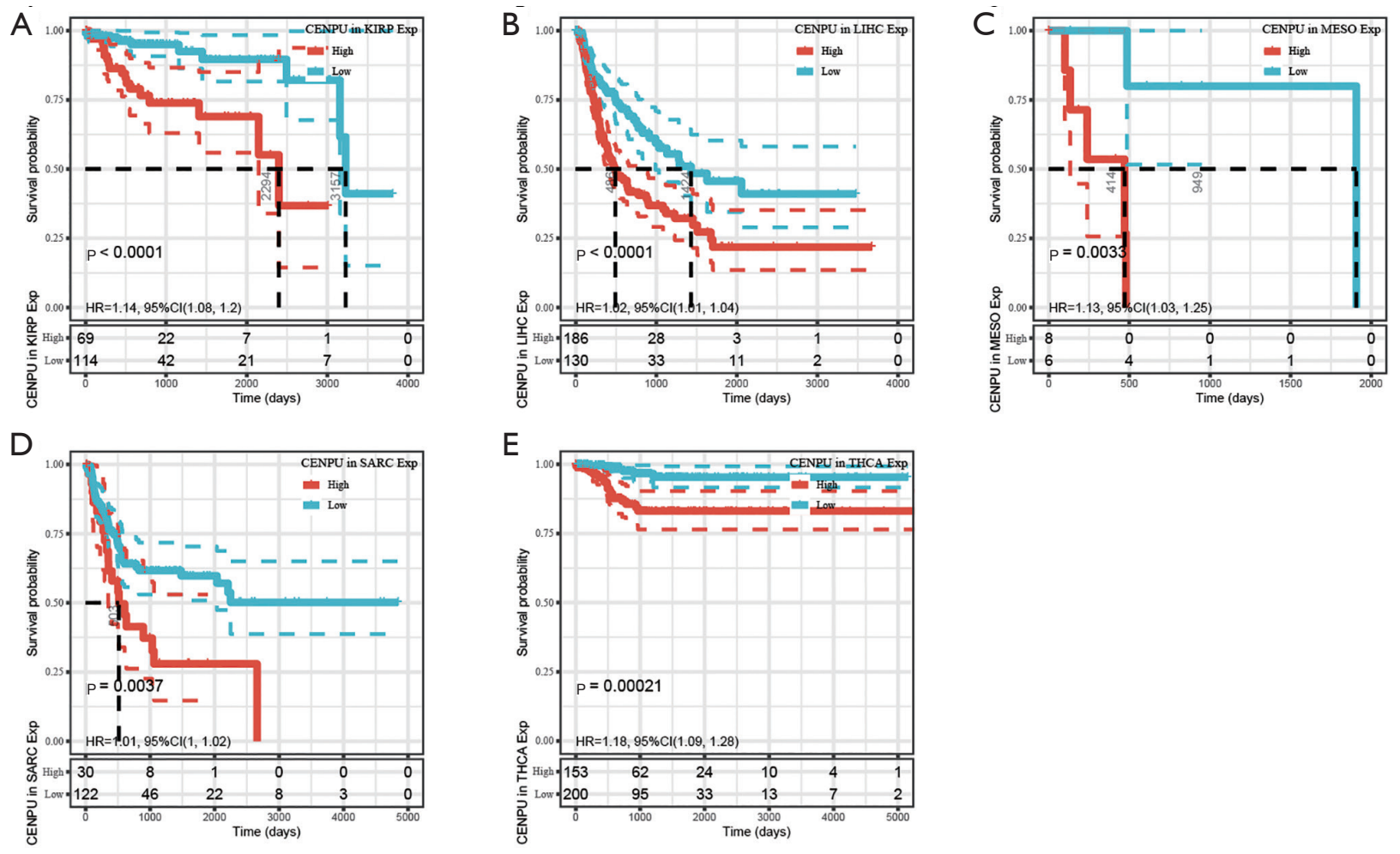

Figure $6 \mathrm{KM}$ analysis between CENP-U expression and DFI in KIRP, LIHC, MESO, SARC and THCA. Data are shown with KM curves. KM, Kaplan-Meier; CENP-U, centromere protein U; DFI, disease-free interval; KIRP, kidney renal papillary cell carcinoma; LIHC, liver hepatocellular carcinoma; MESO, mesothelioma; SARC, sarcoma; THCA, thyroid cancer. 
Type

A

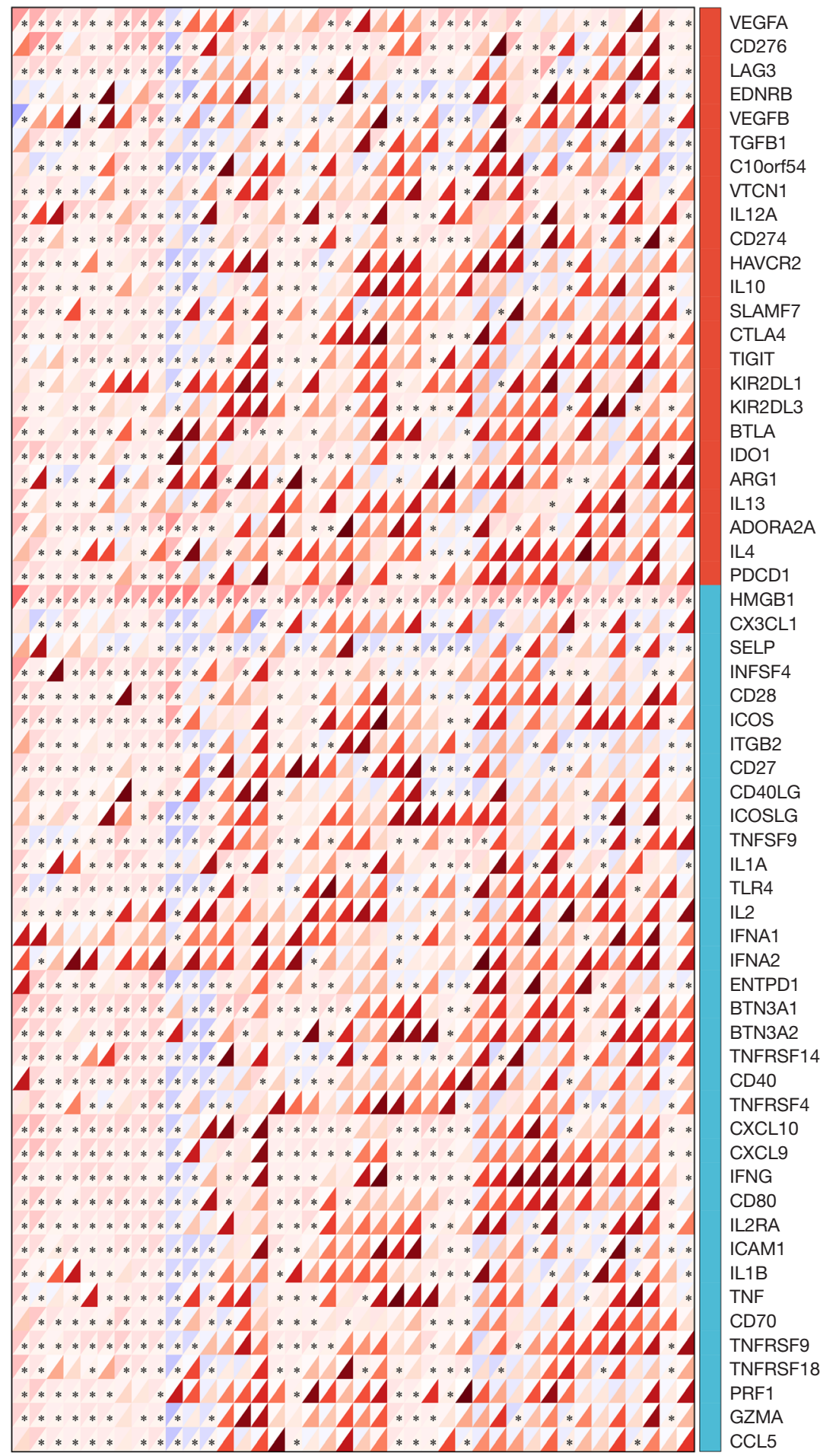

Correlation coefficient

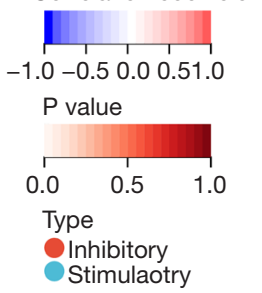

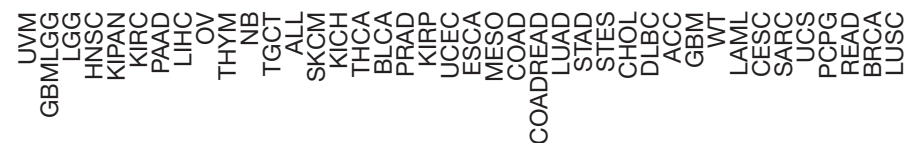


B
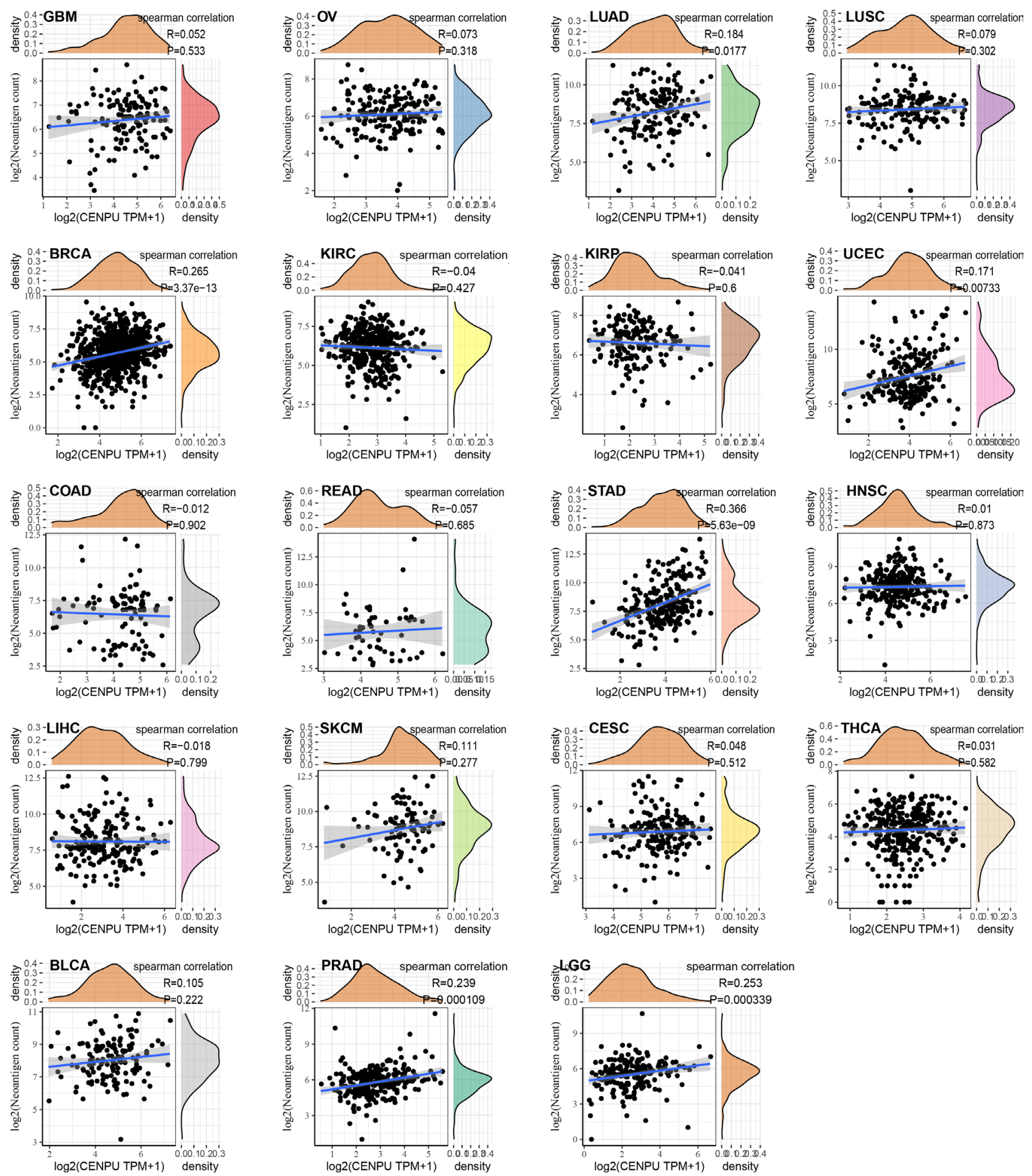

Figure 7 Correlations between CENP-U expression and immune checkpoints and neoantigens. (A) Pan-cancer correlations between $C E N P-U$ expression and 24 immune inhibitors and 36 immune stimulators in. (B) Correlations between $C E N P-U$ expression and number of neoantigens. ${ }^{*} \mathrm{P}<0.05$. CENP-U, centromere protein $\mathrm{U}$. 
A

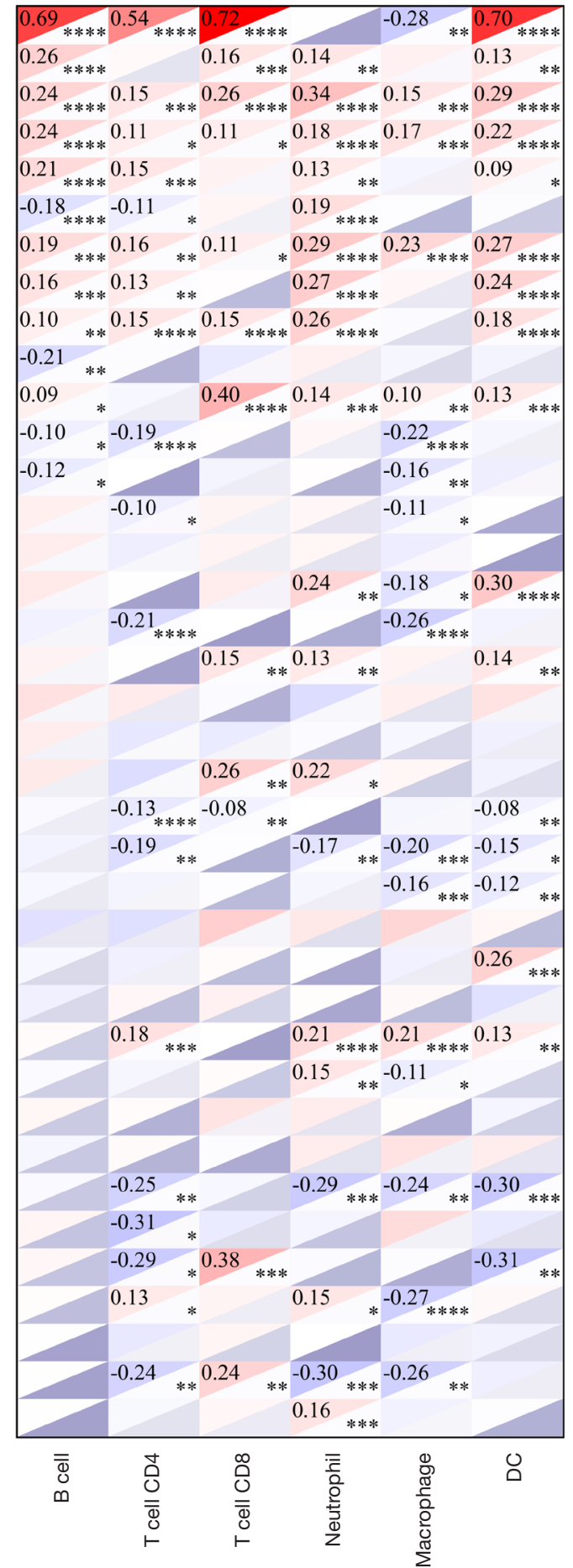

TCGA-THYM $(\mathrm{N}=118)$

TCGA-PRAD $(\mathrm{N}=495)$

TCGA-KIRC ( $N=528)$

TCGA-LGG ( $N=504)$

TCGA-HNSC (N=517)

TCGA-LUAD ( $N=500)$

TCGA-LIHC ( $\mathrm{N}=363)$

TCGA-THCA (N=503)

TCGA-KIPAN ( $\mathrm{N}=878)$

TCGA-ESCA ( $\mathrm{N}=181)$

TCGA-GBMLGG $(\mathrm{N}=656)$

TCGA-STES $(\mathrm{N}=569)$

TCGA-CESC ( $\mathrm{N}=291$ )

TCGA-COADREAD ( $\mathrm{N}=373$ )

TCGA-COAD ( $\mathrm{N}=282)$

TCGA-PAAD ( $\mathrm{N}=177)$

TCGA-STAD ( $N=388)$

TCGA-BLCA (N=405)

TCGA-MESO ( $\mathrm{N}=85)$

TCGA-PCPG $(\mathrm{N}=177)$

TCGA-SKCM-P $(\mathrm{N}=101)$

TCGA-BRCA $(\mathrm{N}=1,077)$

TCGA-SARC ( $\mathrm{N}=258)$

TCGA-LUSC ( $\mathrm{N}=491$ )

TCGA-CHOL ( $\mathrm{N}=36)$

TCGA-UCEC ( $N=178)$

TCGA-UCS $(\mathrm{N}=56)$

TCGA-OV (N=417)

TCGA-SKCM-M ( $\mathrm{N}=351)$

TCGA-ACC (N=77)

TCGA-KICH (N=65)

TCGA-TGCT (N=132)

TCGA-DLBC ( $\mathrm{N}=46)$

TCGA-UVM (N=79)

TCGA-KIRP $(\mathrm{N}=285)$

TCGA-READ ( $\mathrm{N}=91)$

TCGA-GBM $(\mathrm{N}=152)$

TCGA-SKCM (N=452)
Correlation coefficient

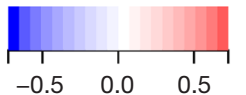

$P$ value

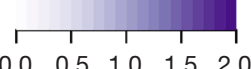



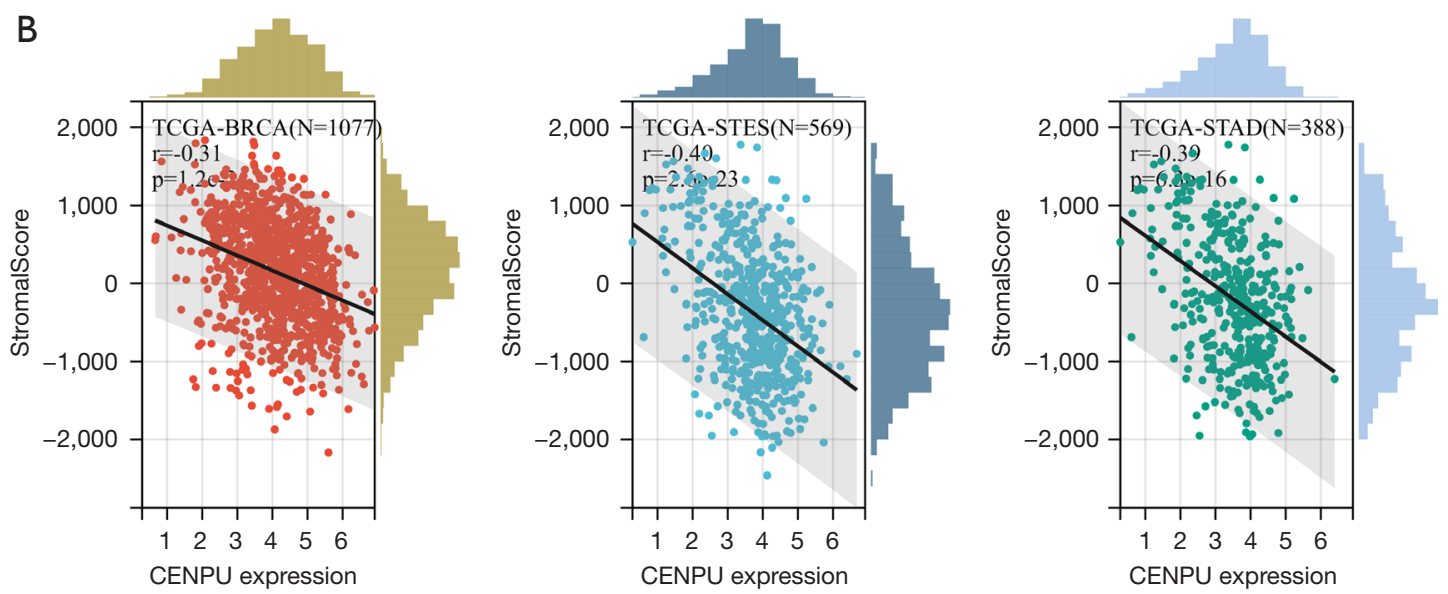

Figure 8 Correlations between $C E N P-U$ expression and tumor-infiltrating immune cells and TME. (A) Pan-cancer correlation analysis across between CENP-U expression and B cells, CD4 ${ }^{+} \mathrm{T}$ cells, CD8 ${ }^{+} \mathrm{T}$ cells, neutrophils, macrophages, and DCs. (B) Representative results of correlation analysis between $C E N P-U$ expression and immune score, stromal score, and ESTIMATE score. ${ }^{*} \mathrm{P}<0.05$; ${ }^{* *} \mathrm{P}<0.01$; ${ }^{* * *} \mathrm{P}<0.001 ;{ }^{* * *} \mathrm{P}<0.0001$. CENP-U, centromere protein $\mathrm{U}$; TME, tumor microenvironment; DC, dendritic cells; ESTIMATE, the Estimation of Stromal and Immune Cells in Malignant Tumors Using Expression data.

myc targets v.1 (Figure 10C), KEGG DNA replication (Figure 10D) and KEGG base excision repair (Figure 10E).

\section{Discussion}

Considerable progress has been achieved in the application of immunotherapy in cancer (2). However, due to the heterogeneity of patients with tumors, the prognosis of a majority of patients still remains poor (4), and thus the development of accurate targeted therapy or multimodal therapy regimens are urgently required. Mitosis-targeted therapy has been successfully used to treat a series of cancers, and recently, the combination of microtubuletargeting agent (MTA) and ICI-based therapy has drawn our attention $(6,33)$.

As a crucial component of kinetochore, CENP-U belongs to the $C E N P-O$ complex, which in turn belongs to CCAN (9) and plays an important role in mitosis. Eliminating of $C E N P-U$ was found to have an influence on both tissue culture cells and mouse development, which suggests the vital role of $C E N P-U$ in mitosis progression $(10,34)$. Additionally, $C E N P-U$ plus Bub1 was found to recruit $P L K 1$ to kinetochore, which is crucial for stabilizing the proper kinetochore-microtubule attachments and in the accurate distribution of DNA $(13,35)$. Recently, elevated $C E N P-U$ expression was identified in a number of cancers (16-24) and high CENP-U expression was correlated to poor prognosis in BRCA, LIHC, BLCA, and LUAD $(16,17,21-23)$. However, the underlying mechanisms remain unclear. Consequently, we conducted a pan-cancer analysis of $C E N P-U$ to evaluate its role in prognosis, immunity, metabolism, and therapeutic potential.

We first performed an analysis of the expression level in normal tissues and a comparison of CENP- $U$ expression between tumor tissues and matched normal tissues. With normal tissues, we identified the highest-level CENP-U expression in hematopoietic organs and the testes and the lowest-level CENP- $U$ expression in muscle and the heart. Taking the mitosis-related role of CENP-U and the proliferative demand of the testes, bone marrow, muscle, and heart into account $(36,37)$, we can assume that the differential expression of $C E N P-U$ in normal tissues confirms the importance of CENP- $U$ in cell cycle progression. High-level expression of $C E N P-U$ in the testes may also account for previous results that $C E N P-U$ expression was elevated in all tumors compared with matched normal tissues except for TGCT. Our results were also consistent with previous findings that showed CENP-U expression to be increased in BRCA (16,17), GBM (18), PRAD (19), osteosarcoma (20), LIHC (21), BLCA (22), LUAD (23), and OV (24). The differential expression of $C E N P-U$ in other tumors warrants further research. We also analyzed the pan-cancer prognostic role of $C E N P-U$ with clinicopathological and $C E N P-U$ expression data. 
A

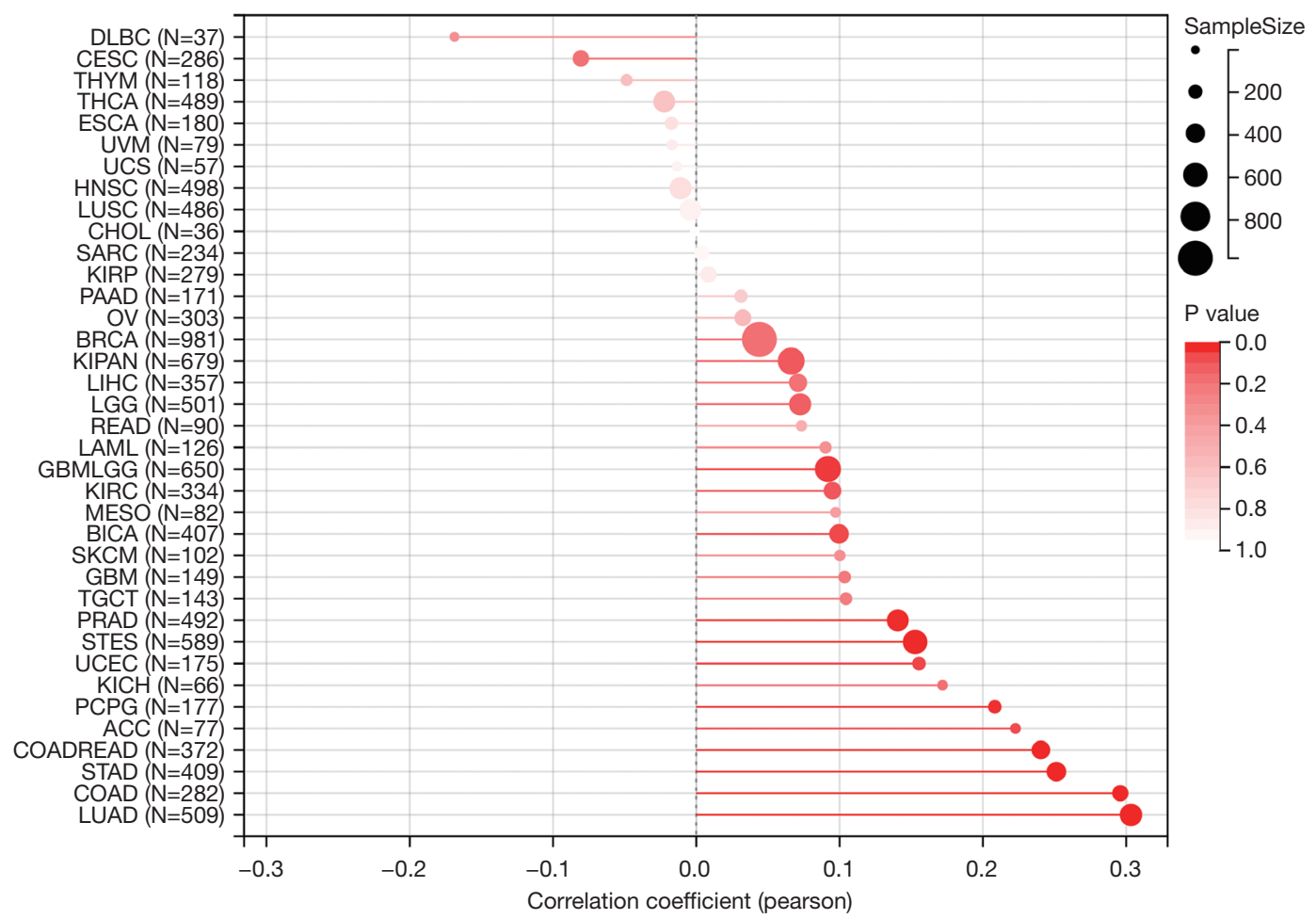

B

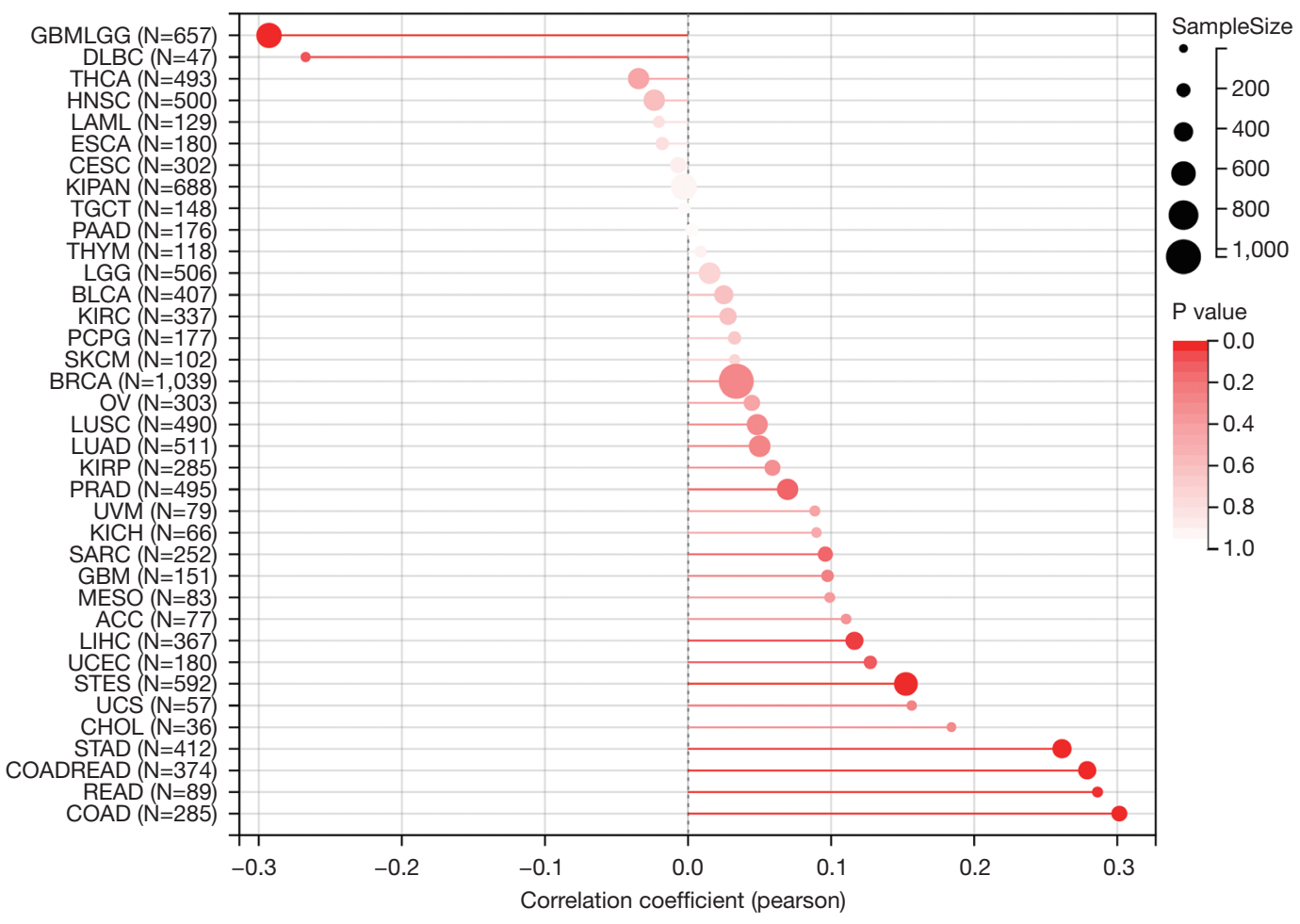

Figure 9 Correlations between CENP-U expression and TMB and MSI. (A) Correlation between $C E N P-U$ expression and TMB. (B) Correlations between $C E N P-U$ expression and MSI. Data are shown with bubble charts. $C E N P-U$, centromere protein U; TMB, tumor mutational burden; MSI, microsatellite instability. 


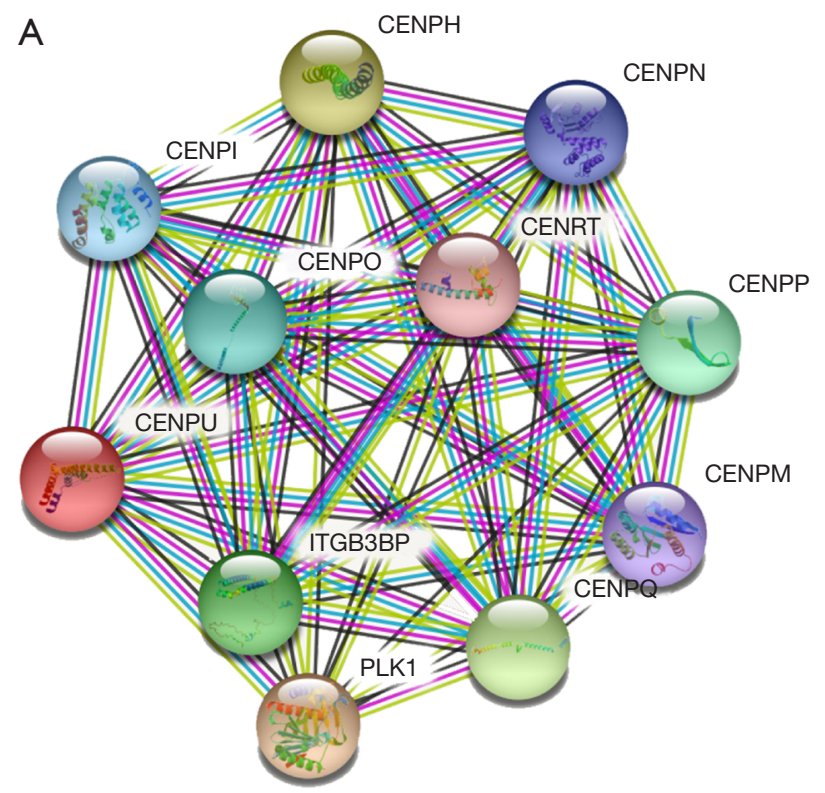

B

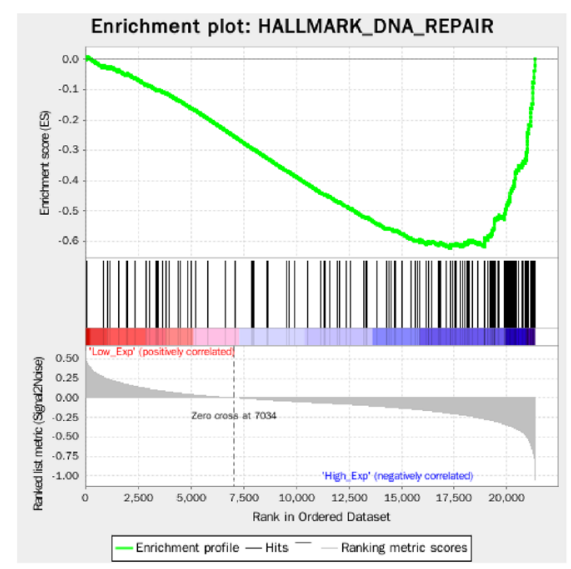

D

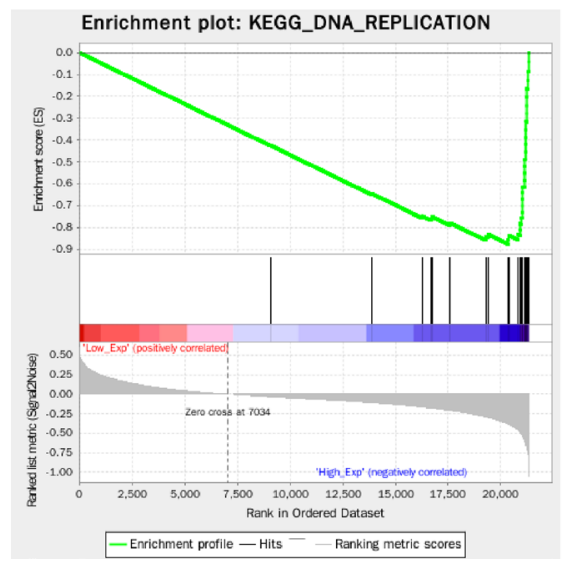

C

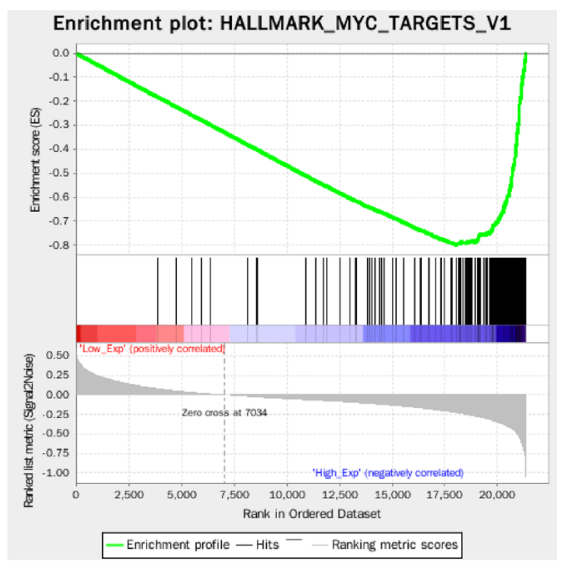

$\mathrm{E}$

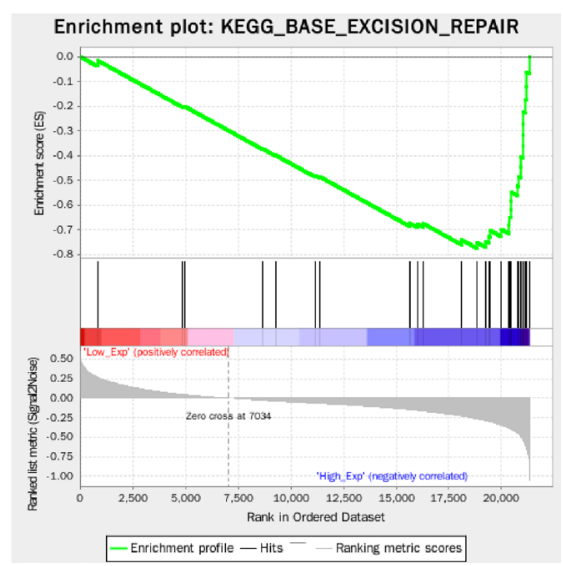

Figure 10 GSEA. (A) Proteins of the $C E N P-U$ function network. (B,C) Representative results of Hallmark analysis of $C E N P-U$. (D,E) Representative results of KEGG analysis of $C E N P-U$. GSEA, gene set enrichment analysis; $C E N P-U$, centromere protein U; KEGG, Kyoto Encyclopedia of Genes and Genomes. 
Similar to previous studies $(16,17,21-23)$, we found that elevated $C E N P-U$ expression was correlated with poor prognosis, as indicated by measures of OS, DSS, DFI, and PFI, across a variety of tumor types. Conversely, high $C E N P-U$ expression was correlated to longer OS in THYM and DLBC, which may be explained by the role of CENP- $U$ in tumor immunity. In summary, CENP- $U$ was found to be differentially expressed in all tumors and may thus serve as a marker for prognosis.

Numerous immune checkpoints have been identified and studied in the past decade, with anti- $P D-1 / P D-L 1$ drugs appearing to be effective therapeutic regimens. However, the heterogeneity of tumor patients means that only a limited number of patients can benefit from this type of therapy (25). In our study, correlation analysis of $C E N P-U$ expression and immune checkpoints was performed. Within 24 immune inhibitors, $C E N P-U$ expression was positively linked to $P D-L 1$ in 21 tumors, to VEGFA in 21 tumors, to CD276 in 25 tumors, and to $L A G 3$ in 21 tumors. Meanwhile, among 36 immune stimulators, CENP-U expression was found to be positively associated with $H M G B 2$ in all 40 tumors, CXCL10 in 22 tumors, CXCL9 in 21 tumors, and TNFSF 4 in 20 tumors, but negatively associated with SELP in 20 tumors. In the immune stimulators, $H M G B 2$ was positively correlated to $C E N P-U$ expression in all 40 tumors. Previous studies have consistently reported $C E N P-U$ to be positively correlated with $H M G B 2$ in OV (24), and it has been shown that knockdown of $C E N P-U$ expression in bladder cancer can decrease the expression of HMGB1 (22). Among other immune checkpoints, (I) the PD-1 and PDL1 axis was shown to act as a mechanism of tumor cell escape from host immunity (38), (II) $L A G 3$ was reported to exert a suppressive effect on activated T cells (39), (III) VEGFA was found to inhibit the trafficking of cytotoxic $\mathrm{T}$ lymphocyte (CTL) and the maturation of DCs (40), (IV) CD276 was recently identified as a $T$ cell inhibitor (41), and (V) CXCL9 and CXCL10 were demonstrated to promote the differentiation of naïve $\mathrm{T}$ cells to $\mathrm{T}$ helper (Th) cells (42); however, (VI) the effect of other immune checkpoints remains relatively controversial. From the abovementioned results, we can surmise that, compared with immune stimulators correlated to $C E N P-U$ expression, the immune inhibitors with correlations had a more profound effect on tumor immunity. Furthermore, from another perspective, we found that there are differences in number between correlated immune stimulators and correlated immune inhibitors in each tumor, which revealed the influence of $C E N P-U$. Taken together, CENP-U has a significant role immune regulation and thus has potential as a therapeutic target.

As the basis of immunotherapy, immune cells of the innate immune system, including DCs, macrophages, neutrophils, mast cells, and monocytes; and adaptive immune system, including $\mathrm{T}$ cells and $\mathrm{B}$ cells, infiltrate the TME and regulate tumor progression $(31,43)$. To further our understanding of the role of CENP- $U$ in immunity, correlation analysis of $C E N P-U$ expression and tumor-infiltrating immune cells with ESTIMATE score was performed. In our study, positive correlations were identified between $C E N P-U$ expression and infiltrated immune cells in KIRC, GBMLGG, PAAD, UVM, while negative correlations were identified between $C E N P-U$ expression and infiltrated immune cells in TGCT, DLBC, and DC. Intriguingly, $C E N P-U$ expression was found to be significantly and positively correlated with $\mathrm{B}$ cells, $\mathrm{CD} 4^{+} \mathrm{T}$ cells, $\mathrm{CD}^{+} \mathrm{T}$ cells, and DCs, which may account for that high $C E N P-U$ expression was associated with longer OS in patients with THYM. In addition, $C E N P-U$ expression was also found to be linked to stromal score in a series of tumors, which suggests that $C E N P-U$ does not only influence the infiltration of immune cells, but may also play an important role in stromal cell infiltration, including in epithelial cells, fibroblasts, and vascular cells, which may affect the purity of tumor (30). Thus, CENP-U holds the potential of being a crucial modulator of immunity, and targeting $C E N P-U$ may be a promising therapeutic strategy.

To improve the effect of immunotherapy, the ability to predict checkpoint inhibitor (CPI) response is essential (26). TMB represents the number of mutations in tumor cells and has recently been identified as an effective predictor of CPI and an important biomarker for identifying patients with potential for immunotherapy in a number of cancers $(26,44,45)$. Furthermore, deficient mismatch repair (dMMR)/MSI status has been widely studied and has been found to contribute to the high efficacy of immunotherapy across a series of tumor types $(27,46)$. In our study, we performed correlation analysis of $C E N P-U$ expression and TMB and MSI to explore the pan-cancer prognostic capacity of $C E N P-U$ in immunotherapy. The results revealed $C E N P-U$ expression to be positively correlated to both TMB and MSI in STAD, COADREAD, and COAD, and other associations were also identified. Overall, CENP-U expression may be a potential predictor of immunotherapy, and further clinical trials are warranted.

To clarify of the diverse functions of CENP- $U$ and glean insights into the role of $C E N P-U$, we performed 
GSEA and obtained the functional network and enriched signaling pathways of CENP-U. The proteins in the functional network were all mitosis-related, including CENP-O, CENP-P, CENP-Q, CENP-R, CENP-I, CENP-H, CENP-M, CENP-N, CENP-T, and PLK1. As a component of kinetochore, CENP-U has been consistently reported to interact with other components of CCAN: (I) CENP-U belongs to the CENP-OPQUR complex, and CENP-Q directly binds to CENP-U (47); (II) CENP-CHIKMLN is required for recruitment of the CENP-OPQUR complex, and $C E N P-U$ interacts with the CENP-H/CENP-I complex $(47,48)$; (III) being more closely located to the $C E N P-A$ nucleosome, CENP-U, CENP-H, CENP-C, CENP-M, $C E N P-N$, and $C E N P-T$ were identified and named the $C E N P-A$ nucleosome-associated complex (NAC) (49); and (IV) in addition to $C E N P-Q$ complex, $C E N P-U$ also binds to some of the $C E N P-A$ NAC components, including $C E N P-H$ and CENP-T (49). This latter finding is in line with our GSEA result in which KEGG DNA replication was highly enriched in the $C E N P-U$ high expression group. Moreover, $P L K 1$ plays an important role in stabilizing kinetochoremicrotubule attachments, ensuring accurate distribution of DNA, and promoting cell cycle progression (49). Studies have reported that PLK1 is recruited to the inner kinetochore by $C E N P-U$ and to the outer kinetochore by $B U B 1$, and $C E N P-U$ may be the sole receptor of PLK1 in the core kinetochore $(13,35,49)$. It has also been found that cells lacking either $C E N P-U$ or $B U B 1$ signaling are more sensitive to $P L K 1$ inhibitor (35). Considering the emergence of ICI therapy plus MTAs (33), the addition of CENP-U inhibitor to this regimen may be even more effective, but more studies are needed to confirm this. Furthermore, our GSEA results showed that $C E N P-U$ was highly expressed in Hallmark DNA repair and KEGG base excision repair signaling pathways. Accumulation of DNA damage can lead to genome instability and ultimately result in the genetic pathogenesis of cancer. DDR is the underlying mechanism by which organisms prevent DNA damage $(50,51)$. Previous studies have indicated that CENP-U has as an essential role in DDR. For one, as a crucial part of the kinetochore and CCAN, and the sole receptor of PLK1 in the core kinetochore, CENP-U strengthens the stability of the kinetochore or spindle and further kinetochore-microtubule attachments by binding to PLK1 $(9,13,35)$. In this way, $C E N P-U$ further ensures the equal distribution of chromosome and prevents the emergence of aneuploidy (52). For another, as demonstrated by Zeitlin et al., CENP-U plus CENP-A,CENP-N, and CENP-T is rapidly recruited to the location of double-strand breaks in DNA (14), which confirms the role of CENP-U in DDR. In summary, as an important component of the kinetochore and a mitosis-related gene, $C E N P-U$ contributes to the stability of the spindle and the accurate distribution of DNA, ultimately promoting cell cycle and tumorigenesis.

In conclusion, our study showed that $C E N P-U$ was differentially expressed across common tumors and it may act as a therapeutic and prognostic biomarker. Limitations to our work is that only bioinformatical analyses of open accessible databases were performed with no experimental data available. Thus, this study provides the foundation for subsequent studies and further studies are needed to verify our findings.

\section{Acknowledgments}

The authors appreciate the academic support from the AME Lung Cancer Collaborative Group.

Funding: This work was supported by the National Natural Science Foundation of China (Nos. 82173252, 81871866), the Shaanxi Social Development Science and Technology Key Project (No. 2016SF-308), and the Project of Tangdu Hospital, The Fourth Military Medical University (2018 Key Talents).

\section{Footnote}

Reporting Checklist: The authors have completed the REMARK reporting checklist. Available at https://dx.doi. org/10.21037/atm-21-6516

Conflicts of Interest: All authors have completed the ICMJE uniform disclosure form (available at https://dx.doi. org/10.21037/atm-21-6516). The authors have no conflicts of interest to declare.

Ethical Statement: The authors are accountable for all aspects of the work in ensuring that questions related to the accuracy or integrity of any part of the work are appropriately investigated and resolved. This study was approved by the Ethics Committee of the Fourth Military Medical University (No. TDLL-KY-202106-09). The study was conducted in accordance with the Declaration of Helsinki (as revised in 2013).

Open Access Statement: This is an Open Access article distributed in accordance with the Creative Commons 
Attribution-NonCommercial-NoDerivs 4.0 International License (CC BY-NC-ND 4.0), which permits the noncommercial replication and distribution of the article with the strict proviso that no changes or edits are made and the original work is properly cited (including links to both the formal publication through the relevant DOI and the license). See: https://creativecommons.org/licenses/by-nc-nd/4.0/.

\section{References}

1. Siegel RL, Miller KD, Fuchs HE, et al. Cancer Statistics, 2021. CA Cancer J Clin 2021;71:7-33. Erratum in: CA Cancer J Clin 2021;71:359.

2. Ge X, Zhang Z, Zhang S, et al. Immunotherapy beyond progression in patients with advanced non-small cell lung cancer. Transl Lung Cancer Res 2020;9:2391-400.

3. Mitchell D, Dey M. Neoadjuvant anti-PD-1 immunotherapy for recurrent glioblastoma. Transl Cancer Res 2019;8:S577-79.

4. Gavrielatou N, Doumas S, Economopoulou P, et al. Biomarkers for immunotherapy response in head and neck cancer. Cancer Treat Rev 2020;84:101977.

5. Hanahan D, Weinberg RA. Hallmarks of cancer: the next generation. Cell 2011;144:646-74.

6. Liu X, Chen Y, Li Y, et al. Targeting mitosis exit: A brake for cancer cell proliferation. Biochim Biophys Acta Rev Cancer 2019;1871:179-91.

7. McKinley KL, Cheeseman IM. The molecular basis for centromere identity and function. Nat Rev Mol Cell Biol 2016;17:16-29.

8. Cheeseman IM, Desai A. Molecular architecture of the kinetochore-microtubule interface. Nat Rev Mol Cell Biol 2008;9:33-46.

9. Perpelescu M, Fukagawa T. The ABCs of CENPs. Chromosoma 2011;120:425-46.

10. Kagawa N, Hori T, Hoki Y, et al. The CENP-O complex requirement varies among different cell types. Chromosome Res 2014;22:293-303.

11. Hozé C, Escouflaire C, Mesbah-Uddin M, et al. Short communication: A splice site mutation in CENPU is associated with recessive embryonic lethality in Holstein cattle. J Dairy Sci 2020;103:607-12.

12. Kang YH, Park JE, Yu LR, et al. Self-regulated Plk1 recruitment to kinetochores by the Plk1-PBIP1 interaction is critical for proper chromosome segregation. Mol Cell 2006;24:409-22.

13. Singh P, Pesenti ME, Maffini S, et al. BUB1 and CENP-U, Primed by CDK1, Are the Main PLK1 Kinetochore
Receptors in Mitosis. Mol Cell 2021;81:67-87.e9.

14. Zeitlin SG, Baker NM, Chapados BR, et al. Double-strand DNA breaks recruit the centromeric histone CENP-A. Proc Natl Acad Sci U S A 2009;106:15762-7.

15. Wang J, Liu X, Chu HJ, et al. Centromere Protein I (CENP-I) Is Upregulated in Gastric Cancer, Predicts Poor Prognosis, and Promotes Tumor Cell Proliferation and Migration. Technol Cancer Res Treat 2021;20:15330338211045510.

16. Yang F, Wang YH, Dong SY, et al. MLF1IP promotes cells proliferation and apoptosis by regulating CyclinD1 in breast cancer. Int J Clin Exp Pathol 2017;10:11554-62.

17. Pan T, Zhou D, Shi $Z$, et al. Centromere protein $U$ (CENPU) enhances angiogenesis in triple-negative breast cancer by inhibiting ubiquitin-proteasomal degradation of COX-2. Cancer Lett 2020;482:102-11.

18. Hanissian SH, Teng B, Akbar U, et al. Regulation of myeloid leukemia factor-1 interacting protein (MLF1IP) expression in glioblastoma. Brain Res 2005;1047:56-64.

19. Zhang L, Ji G, Shao Y, et al. MLF1 interacting protein: a potential gene therapy target for human prostate cancer? Med Oncol 2015;32:454.

20. Li Q, Liang J, Chen B. Identification of CDCA8, DSN1 and BIRC5 in Regulating Cell Cycle and Apoptosis in Osteosarcoma Using Bioinformatics and Cell Biology. Technol Cancer Res Treat 2020;19:1533033820965605.

21. Cui Z, Xiao L, Chen F, et al. High mRNA Expression of CENPL and Its Significance in Prognosis of Hepatocellular Carcinoma Patients. Dis Markers 2021;2021:9971799.

22. Wang S, Liu B, Zhang J, et al. Centromere protein $\mathrm{U}$ is a potential target for gene therapy of human bladder cancer. Oncol Rep 2017;38:735-44.

23. Li J, Wang ZG, Pang LB, et al. Reduced CENPU expression inhibits lung adenocarcinoma cell proliferation and migration through PI3K/AKT signaling. Biosci Biotechnol Biochem 2019;83:1077-84.

24. Li H, Zhang H, Wang Y. Centromere protein U facilitates metastasis of ovarian cancer cells by targeting high mobility group box 2 expression. Am J Cancer Res 2018;8:835-51.

25. He X, Xu C. Immune checkpoint signaling and cancer immunotherapy. Cell Res 2020;30:660-9.

26. Litchfield K, Reading JL, Puttick C, et al. Meta-analysis of tumor- and $\mathrm{T}$ cell-intrinsic mechanisms of sensitization to checkpoint inhibition. Cell 2021;184:596-614.e14.

27. Le DT, Durham JN, Smith KN, et al. Mismatch repair deficiency predicts response of solid tumors to PD-1 
blockade. Science 2017;357:409-13.

28. Barretina J, Caponigro G, Stransky N, et al. The Cancer Cell Line Encyclopedia enables predictive modelling of anticancer drug sensitivity. Nature 2012;483:603-7.

29. Li T, Fan J, Wang B, et al. TIMER: A Web Server for Comprehensive Analysis of Tumor-Infiltrating Immune Cells. Cancer Res 2017;77:e108-10.

30. Yoshihara K, Shahmoradgoli M, Martínez E, et al. Inferring tumour purity and stromal and immune cell admixture from expression data. Nat Commun 2013;4:2612.

31. Zhang $Y$, Zhang $Z$. The history and advances in cancer immunotherapy: understanding the characteristics of tumor-infiltrating immune cells and their therapeutic implications. Cell Mol Immunol 2020;17:807-21.

32. Yates LR, Campbell PJ. Evolution of the cancer genome. Nat Rev Genet 2012;13:795-806.

33. Serpico AF, Visconti R, Grieco D. Exploiting immunedependent effects of microtubule-targeting agents to improve efficacy and tolerability of cancer treatment. Cell Death Dis 2020;11:361.

34. Hori T, Okada M, Maenaka K, et al. CENP-O class proteins form a stable complex and are required for proper kinetochore function. Mol Biol Cell 2008;19:843-54.

35. Chen Q, Zhang M, Pan X, et al. Bub1 and CENP-U redundantly recruit $\mathrm{Plk} 1$ to stabilize kinetochoremicrotubule attachments and ensure accurate chromosome segregation. Cell Rep 2021;36:109740.

36. Bentzinger CF, Wang YX, von Maltzahn J, et al. The emerging biology of muscle stem cells: implications for cell-based therapies. Bioessays 2013;35:231-41.

37. Porter KE, Turner NA. Cardiac fibroblasts: at the heart of myocardial remodeling. Pharmacol Ther 2009;123:255-78.

38. Gong J, Chehrazi-Raffle A, Reddi S, et al. Development of PD-1 and PD-L1 inhibitors as a form of cancer immunotherapy: a comprehensive review of registration trials and future considerations. J Immunother Cancer 2018;6:8.

39. Maçon-Lemaître L, Triebel F. The negative regulatory function of the lymphocyte-activation gene-3 co-receptor (CD223) on human T cells. Immunology 2005;115:170-8.

40. Fukumura D, Kloepper J, Amoozgar Z, et al. Enhancing cancer immunotherapy using antiangiogenics: opportunities and challenges. Nat Rev Clin Oncol 2018;15:325-40.

41. Liu S, Liang J, Liu Z, et al. The Role of CD276 in
Cancers. Front Oncol 2021;11:654684.

42. Tokunaga R, Zhang W, Naseem M, et al. CXCL9, CXCL10, CXCL11/CXCR3 axis for immune activation - A target for novel cancer therapy. Cancer Treat Rev 2018;63:40-7.

43. Adams JL, Smothers J, Srinivasan R, et al. Big opportunities for small molecules in immuno-oncology. Nat Rev Drug Discov 2015;14:603-22.

44. Carbone DP, Reck M, Paz-Ares L, et al. First-Line Nivolumab in Stage IV or Recurrent Non-Small-Cell Lung Cancer. N Engl J Med 2017;376:2415-26.

45. Hugo W, Zaretsky JM, Sun L, et al. Genomic and Transcriptomic Features of Response to Anti-PD-1 Therapy in Metastatic Melanoma. Cell 2016;165:35-44.

46. Berger MF, Hodis E, Heffernan TP, et al. Melanoma genome sequencing reveals frequent PREX2 mutations. Nature 2012;485:502-6.

47. Pesenti ME, Prumbaum D, Auckland P, et al. Reconstitution of a 26-Subunit Human Kinetochore Reveals Cooperative Microtubule Binding by CENPOPQUR and NDC80. Mol Cell 2018;71:923-39.e10.

48. Minoshima Y, Hori T, Okada M, et al. The constitutive centromere component CENP-50 is required for recovery from spindle damage. Mol Cell Biol 2005;25:10315-28.

49. Kang YH, Park CH, Kim TS, et al. Mammalian polo-like kinase 1-dependent regulation of the PBIP1-CENP-Q complex at kinetochores. J Biol Chem 2011;286:19744-57.

50. Le DT, Uram JN, Wang H, et al. PD-1 Blockade in Tumors with Mismatch-Repair Deficiency. N Engl J Med 2015;372:2509-20.

51. Kang C, Xu Q, Martin TD, et al. The DNA damage response induces inflammation and senescence by inhibiting autophagy of GATA4. Science 2015;349:aaa5612.

52. Ohashi A, Ohori M, Iwai K, et al. Aneuploidy generates proteotoxic stress and DNA damage concurrently with p53-mediated post-mitotic apoptosis in SAC-impaired cells. Nat Commun 2015;6:7668.

(English Language Editor: J. Gray)

Cite this article as: Shao C, Wang Y, Duan H, Ding P, Zhang Y, Ning J, Han J, Jiang T, Yan X. Mitosis-related gene CENP-U as a potential biomarker in malignancy. Ann Transl Med 2021;9(23):1744. doi: 10.21037/atm-21-6516 